\title{
An algorithm for Mean Curvature Motion
}

\author{
Antonin Chambolle ${ }^{\dagger}$ \\ CEREMADE (CNRS UMR 7534), Université de Paris-Dauphine, 75775 Paris CEDEX 16, France
}

[Received 2 July 2003 and in revised form 25 December 2003]

\begin{abstract}
We propose a new algorithm for computing the evolution by mean curvature of a hypersurface. Our algorithm is a variant of the variational approach of Almgren, Taylor and Wang [2]. We show that it approximates, as the time step goes to zero, the generalized motion (in the sense of barriers or viscosity solutions). The results still hold for the Anisotropic Mean Curvature Motion, as long as the anisotropy is smooth.
\end{abstract}

\section{Introduction}

In this paper, we propose a method for computing the evolution $E(t)$ of a volume in $\mathbb{R}^{N}$ whose boundary moves with a velocity equal to its mean curvature (in the normal direction). We also consider the situation where the velocity is a function of the curvature tensor, corresponding to the first variation of the "anisotropic" perimeter $\int_{\partial E} \varphi^{\circ}\left(\nu_{E}\right) \mathrm{d} \mathcal{H}^{N-1}$, where $\nu_{E}$ is the normal to $\partial E$ and $\varphi^{\circ}$ is a convex 1-homogeneous function. This kind of motion belongs to the class of so-called "geometric" evolutions.

Classical (smooth) solutions to such evolution equations are shown to exist only up to some finite time, past which they usually either disappear or form singularities. We refer to [34] for a proof of existence of classical solutions for very general geometric motions, and to [2] for a proof in the cases which are considered in this paper (see also [4]).

Among the attempts to understand the behaviour of solutions past singularities, various definitions of generalized motion have been proposed. Apart from the approaches of Brakke [19] and of Almgren, Taylor and Wang [2] and Luckhaus and Sturzenhecker [45], all other approaches, to our knowledge, are based on an inclusion principle which is the geometrical equivalent of the maximum principle for PDEs. It is well known that the Mean Curvature Motion is "monotone", in the following sense: if two solids $E(t)$ and $F(t)$ evolve smoothly by Mean Curvature Motion for $t \in\left(t_{0}, t_{1}\right)$, then if $E\left(t_{0}\right) \subset F\left(t_{0}\right)$, this property is conserved for all subsequent times, and $E\left(t_{1}\right) \subset F\left(t_{1}\right)$. This observation leads to two different (but equivalent) theories for a generalized motion.

A first idea, of Osher and Sethian [48], is to consider the evolving hypersurface as the level set of a function $u$, and to find the corresponding PDE for $u$. It is not difficult to find that $u(t, x)$ must solve the following degenerate elliptic equation:

$$
\frac{\partial u}{\partial t}=|\nabla u| \operatorname{div} \frac{\nabla u}{|\nabla u|} .
$$

It is then shown that if $u_{0}$ is bounded and uniformly continuous, then equation (1) has a unique viscosity solution, and all level sets of $u(t, x)$ evolve according to a motion that coincides with the Mean Curvature Motion for smooth evolutions, and satisfies the inclusion principle. This problem

${ }^{\dagger}$ Email: antonin.chambolle@ ceremade.dauphine.fr 
has been studied by Evans and Spruck [28-31], and, for more general geometric evolution equations, by Chen, Giga and Goto [22]. We refer to [23] for the general theory of viscosity solutions of second order elliptic and parabolic PDEs, to [6] for the theory of discontinuous solutions, as well as to [8] in the particular case of geometric motions.

Another approach to defining a generalized evolution is the notion of "minimal barriers" of De Giorgi. The idea is to define the generalized motion of a set by comparison with smooth evolutions inside and outside the evolving set. This approach has been studied in particular by Bellettini, Novaga and Paolini [17, 11, 12, 14]. It is essentially equivalent to the viscosity approach, as shown in [16, 13], but it seems sometimes more natural in the context of this paper.

On the other hand, Almgren, Taylor and Wang have proposed in [2] a variational approach to constructing evolutions at all times. The same approach has been simultaneously proposed, in the isotropic case, by Luckhaus and Sturzenhecker [45]. The idea of these authors follows the framework of "Minimizing Movements" introduced by De Giorgi and described by Ambrosio in [3] (with in particular a small chapter dedicated to Almgren, Taylor and Wang's paper). It is based on a discretization in time and on a minimization problem for computing the surface at time $(k+1) h$ from the surface at time $k h, k \in \mathbb{N}, h>0$. The main drawback in their construction is the lack of uniqueness of the solution of this minimization problem, and of the evolution, given an initial state; this is related to the absence of comparison principle in their construction. On the other hand, they are able to show the consistency of their evolution with the Mean Curvature Motion as long as it remains smooth.

We propose here an approach that unifies the previous constructions. It is based on a new algorithm which is relatively easy to implement, and provides a monotonous selection of Almgren, Taylor and Wang's evolution. The monotonicity, together with the consistency with smooth evolutions proved in [2], allows us to conclude that our algorithm is an approximation of the generalized motion, in the sense of minimal barriers or viscosity solutions. The proof of convergence of our algorithm relies on the formalism of minimal barriers described in [13], which seemed to us more suitable to tackle the problem, given the consistency result of [2]. However, it is very likely that the setting of viscosity solutions and the approaches of Barles and Souganidis [9] and Leoni [44] would have led to the same results.

Our algorithm is similar to other approaches that alternate the resolution of a PDE (or convolution with a smoothing kernel) and truncation, following an initial idea of Merriman, Bence and Osher [46, 47] (see [25, 42, 7, 20, 44, 43]). We point out however that our method, although probably slightly more complicated than these approaches, is valid also in the case of the "Anisotropic" Mean Curvature Motion, which is also considered in [2], as long as the anisotropy remains "smooth" (in a sense made precise later on). Some sort of anisotropic diffusion-generated curvature motion is introduced in [51] but it is different from what is usually understood by Anisotropic Mean Curvature Motion. We refer to [41, 55] for the physical motivations of this motion. We show that a simple anisotropic version of our algorithm converges to the generalized motion by anisotropic mean curvature (we follow the definition in [18]), defined as before in the sense of barriers or viscosity solutions. The proof is again based on the monotonicity of our algorithm combined with the consistency of [2].

We have to mention other approaches to computing anisotropic curvature flows, which rely on an Allen-Cahn approximation of the flow (see for instance [27, 49, 32]).

Of particular interest is the situation where the anisotropy is nonsmooth, that is, when the above-mentioned convex function $\varphi^{\circ}$ is nondifferentiable (just Lipschitz). This leads to the so-called 
"Crystalline Curvature Motion" [54, 53, 5]. In this case, the equation becomes nonlocal and little is known. In dimension two, however, a principle of comparison is shown in [35] and M.-H. Giga and Y. Giga can define a generalized evolution (see [33] and the references herein), based on a variant of the classical viscosity solutions. It is likely that our algorithm still converges to this generalized motion, since in the planar crystalline case, Almgren and Taylor [1] still can show the consistency of the "flat curvature flow" with the crystalline motion; however, we have not investigated this situation in the present paper. In dimension three, Giga, Gurtin and Matias [36] consider a movement of crystals in which all facets evolve with uniform velocity. This movement should not coincide with the crystalline motion for all initial shapes. They show short-time existence and a principle of inclusion but no notion of generalized motion is known. A major difficulty in the study of crystalline curvature motion comes from the phenomenon of "facet-breaking" pointed out in [15], and which is not taken into account in [36]. We conjecture that our selection of Almgren, Taylor and Wang's algorithm still converges to a reasonable evolution. Other algorithms for computing crystalline flows in 2D are proposed by Girão and Kohn [37-39]. A proof of convergence of an Allen-Cahn approximation is found in [10].

\section{A new algorithm}

\subsection{Description}

Throughout the paper, $\Omega$ is a bounded open subset of $\mathbb{R}^{N}$. All that follows can easily be adapted to the case $\Omega=\mathbb{T}^{N}=(\mathbb{R} / \mathbb{Z})^{N}$, which is also interesting. Let $J(u ; \Omega)$ denote the total variation of the function $u \in L_{\text {loc }}^{1}(\Omega)$ :

$$
J(u ; \Omega)=\sup \left\{\int_{\Omega} u(x) \operatorname{div} \xi(x) \mathrm{d} x: \xi \in C_{c}^{1}\left(\Omega ; \mathbb{R}^{d}\right),|\xi(x)| \leqslant 1 \forall x \in \Omega\right\},
$$

with $|\cdot|$ the Euclidean norm in $\mathbb{R}^{N}$. It is clearly a convex and lower semicontinuous functional (as a supremum of continuous linear functionals). It is well known that $J(u ; \Omega)<+\infty$ if and only if the distributional derivative $D u$ of $u$ is a finite Radon measure in $\Omega$, in which case we have $J(u ; \Omega)=|D u|(\Omega)$ and we say that $u$ has bounded variation in $\Omega$. The space of functions $u \in L^{1}(\Omega)$ with $J(u ; \Omega)<+\infty$ is denoted by $B V(\Omega)$. We refer to [40], or to [26, 57], for the properties of $J$ and of functions with bounded variation. Throughout the paper, when no ambiguity can arise, we will often write $J(u)$ instead of $J(u ; \Omega)$.

For $E \subset \Omega$, we define the signed distance $d_{E}$ by

$$
d_{E}(x)=d(x, E)-d\left(x, \mathbb{R}^{N} \backslash E\right) .
$$

Here, $E^{c}$ denotes the set $\Omega \backslash E$ and $d(x, E)=\inf _{y \in E}|x-y|$. It is clear that $\bar{E}=\left\{d_{E} \leqslant 0\right\}$ while $\stackrel{\circ}{E}=\left\{d_{E}<0\right\}$. Let $h>0$ and $w$ be the unique solution of

$$
\min _{w \in L^{2}(\Omega)} \int_{\Omega} \frac{\left(w(x)-d_{E}(x)\right)^{2}}{2 h} \mathrm{~d} x+J(w ; \Omega) .
$$

We define the transformation $T_{h}: \mathcal{P}(\Omega) \rightarrow \mathcal{P}(\Omega)$ by letting $T_{h}(E)=\{w<0\}=\{x \in \Omega$ : $w(x)<0\}$. We also define $T_{h}^{\prime}(E)=\{w \leqslant 0\}$; unless otherwise mentioned, all the statements we will make about $T_{h}$ are also valid for $T_{h}^{\prime}$. 
Given $E_{0} \subset \Omega$ and $h>0$, we define, for every $t>0$,

$$
E^{h}(t)=\left(T_{h}\right)^{[t / h]}\left(E_{0}\right)
$$

where $[\cdot]$ is the integer part. We claim that $\partial E^{h}(t)$ is a time-discrete approximation of the Mean Curvature Motion starting from $\partial E_{0}$. The reason for which it is reasonable to believe so is that if $\nabla w$ is not zero in the neighbourhood of $x \in \Omega$, then near $x$ the Euler equation for $(3)$ is

$$
\frac{w-d_{E}}{h}-\operatorname{div} \frac{\nabla w}{|\nabla w|}=0
$$

It turns out that the expression $\operatorname{div}(\nabla w /|\nabla w|)(x)$ is the mean curvature at $x$ of the level hypersurface $\{w=w(x)\}$. Hence, if $x \in \partial T_{h}(E)$, then (for $w$ continuous)

$$
d_{E}(x)=-h \operatorname{curv}_{\partial T_{h}(E)}(x) .
$$

If $x_{0}$ is the projection of $x$ on $\partial E$ and $v\left(x_{0}\right)=\nabla d_{E}\left(x_{0}\right)$ is the exterior normal to the set $E$ at $x_{0}$, one has $x=x_{0}+d_{E}(x) v\left(x_{0}\right)$. The equation then reads

$$
x=x_{0}-h \operatorname{curv}_{\partial T_{h}(E)}(x) v\left(x_{0}\right) .
$$

This is clearly a discretization for the Mean Curvature Motion, with time step $h$. If $E$ is locally convex around $x_{0}$, and if we assume that also $T_{h}(E)$ is convex near $x$, then $\operatorname{curv}_{\partial T_{h}(E)}(x)$ is positive and the movement goes in the direction opposite to the outer normal to $E$, that is, towards the interior. This is the expected behaviour.

Let us consider the special case where $\Omega=B(0, R)$ and $E=B(0, r)$, with $0<r<R$. Then $d_{E}(x)=|x|-r$ and $w(x)=|x|-r+h(N-1) /|x|$, except near $|x|=0$ and $|x|=R$. If $h$ is small enough, one checks that $\{w=0\}=\{|x|=r(1+\sqrt{1-4 h(N-1) / r}) / 2\}$. If we fix $\varepsilon$ and take $h$ small enough, we get $r-(1+\varepsilon) h(N-1) / r \leqslant|x| \leqslant r-h(N-1) / r$ on $\partial T_{h}(E)$, and this estimate is locally uniform in $r$. One easily deduces that as $h \rightarrow 0$, the limit of the motion $E^{h}(t)$ starting from a ball $E_{0}=B\left(0, r_{0}\right)$ is $B(0, r(t))$, with $\dot{r}(t)=-(N-1) / r(t)$, that is, $r(t)=\sqrt{r_{0}^{2}-2(N-1) t}$ if $t \leqslant r_{0}^{2} /(2(N-1))$ and 0 if $t$ is larger. This happens to be the solution of the Mean Curvature Motion starting from $E_{0}$.

\subsection{Two essential properties}

2.2.1 The algorithm is monotone. Let us now state one of the two key properties of the operator $T_{h}$.

LEMMA 2.1 If $E \subseteq E^{\prime}$, then $T_{h}(E) \subseteq T_{h}\left(E^{\prime}\right)$.

Proof. The proof is quite straightforward. If $E \subseteq E^{\prime}$, then $d_{E} \geqslant d_{E^{\prime}}$. One checks that the mapping $d_{E} \mapsto w$ given by the solution of (3) is monotone (see the sketch of proof below). Hence, $w \geqslant w^{\prime}$ (where $w^{\prime}$ is the solution of 3 ) with $d_{E^{\prime}}$ ). We deduce that $\{w<0\} \subseteq\left\{w^{\prime}<0\right\}$, which proves the lemma.

It remains to show that whenever $f \leqslant g$, the minimizer $w_{f}$ of $\int_{\Omega}|w-f|^{2} /(2 h)+J(w)$ is less than the minimizer $w_{g}$ of $\int_{\Omega}|w-g|^{2} /(2 h)+J(w)$. From the inequalities

$$
\frac{1}{2 h} \int_{\Omega}\left|w_{f} \wedge w_{g}(x)-f(x)\right|^{2} \mathrm{~d} x+J\left(w_{f} \wedge w_{g}\right) \geqslant \frac{1}{2 h} \int_{\Omega}\left|w_{f}(x)-f(x)\right|^{2} \mathrm{~d} x+J\left(w_{f}\right),
$$


$\frac{1}{2 h} \int_{\Omega}\left|w_{f} \vee w_{g}(x)-g(x)\right|^{2} \mathrm{~d} x+J\left(w_{f} \vee w_{g}\right) \geqslant \frac{1}{2 h} \int_{\Omega}\left|w_{g}(x)-g(x)\right|^{2} \mathrm{~d} x+J\left(w_{g}\right)$,

and (see [40])

$$
J\left(w_{f} \wedge w_{g}\right)+J\left(w_{f} \vee w_{g}\right) \leqslant J\left(w_{f}\right)+J\left(w_{g}\right)
$$

one deduces easily that $\int_{\left\{w_{f}>w_{g}\right\}}\left(w_{f}-w_{g}\right)(f-g) \geqslant 0$. Hence if $f<g$, then $w_{f} \leqslant w_{g}$ a.e. in $\Omega$. By continuity of the mapping $f \mapsto w_{f}$ (in $L^{2}(\Omega)$; indeed, if $f_{1}, f_{2} \in L^{2}(\Omega)$, taking the difference of the Euler equations for $w_{f_{1}}$ and $w_{f_{2}}$ and multiplying by $w_{f_{1}}-w_{f_{2}}$ one finds easily that $\left.\left\|w_{f_{1}}-w_{f_{2}}\right\|_{L^{2}(\Omega)} \leqslant\left\|f_{1}-f_{2}\right\|_{L^{2}(\Omega)}\right)$, this remains true when $f \leqslant g$.

2.2.2 The algorithm implements Almgren, Taylor and Wang's flat curvature flow. We now show that our method is just another way to compute the algorithm introduced by Almgren, Taylor and Wang in [2] and by Luckhaus and Sturzenhecker in [45]. Another nice presentation of the approach is given by Ambrosio in [3]. In [2], the evolution of a finite-perimeter set $E \subset \Omega$ across a time step $h>0$ is computed by solving the problem

$$
\min _{F} J(F ; \Omega)+\frac{1}{h} \int_{F \triangle E}\left|d_{E}(x)\right| \mathrm{d} x .
$$

Here, $J(F ; \Omega)$ is a simplified notation for $J\left(\mathbf{1}_{F} ; \Omega\right)$, where $\mathbf{1}_{F}(x)=1$ if $x \in F, 0$ otherwise, is the indicator function of $F$. The minimum is taken over all subsets $F$ of $\Omega$ with finite perimeter, that is, such that $J(F)<+\infty$, and $F \triangle E$ denotes the symmetric difference $(F \backslash E) \cup(E \backslash F)$. In [2], $\Omega=\mathbb{R}^{N}$ (while in our problem the energy becomes unbounded if $\Omega=\mathbb{R}^{N}$ ). We have the following proposition.

Proposition 2.2 The set $T_{h}(E)$ is a solution of (5).

Before proving this result, let us recall some properties of $J$ that are standard facts in convex analysis. We refer to [24] for a good introduction to convex analysis. Here $J$ is viewed as a convex 1.s.c. functional mapping $L^{2}(\Omega)$ to $[0,+\infty]$. Given any convex functional $J$ over the Hilbert space $L^{2}(\Omega)$, one defines its subgradient $\partial J(u)$ at $u$ by

$$
\partial J(u)=\left\{p \in L^{2}(\Omega): J(v) \geqslant J(u)+\int_{\Omega} p(v-u) \forall v \in L^{2}(\Omega)\right\} .
$$

One also defines the Legendre-Fenchel transform $J^{*}$ which is the convex and 1.s.c. function given by

$$
J^{*}(p)=\sup _{u \in L^{2}(\Omega)} \int_{\Omega} p(x) u(x) \mathrm{d} x-J(u) .
$$

It is well known that $J^{* *}$ is the convex 1.s.c. envelope of the functional $J$, in particular, $J^{* *}=J$ whenever $J$ is already l.s.c. and convex. In our case, $J$ is also 1 -homogeneous, that is, $J(t u)=$ $t J(u)$ for all $u$ and all $t>0$. One deduces easily that $J^{*}(p)$ is the characteristic function of a closed and convex set $K \subset L^{2}(\Omega)$ :

$$
J^{*}(p)=\chi_{K}(p)= \begin{cases}0 & \text { if } p \in K \\ +\infty & \text { otherwise }\end{cases}
$$

where the set $K$ is $\left\{p: \int_{\Omega} p u \leqslant J(u) \forall u\right\}=\partial J(0)$. 
The identity $J^{* *}=J$ yields

$$
J(u)=\sup _{p \in K} \int_{\Omega} p(x) u(x) \mathrm{d} x
$$

From the definition 2, one deduces that $K$ is the closure in $L^{2}(\Omega)$ of

$$
\left\{\operatorname{div} \xi: \xi \in C_{c}^{1}\left(\Omega ; \mathbb{R}^{N}\right),|\xi(x)| \leqslant 1 \forall x \in \Omega\right\} .
$$

Another well known identity is the Fenchel identity:

$$
p \in \partial J(u) \Leftrightarrow u \in \partial J^{*}(p) \Leftrightarrow J(u)+J^{*}(p)=\int_{\Omega} p(x) u(x) \mathrm{d} x
$$

from which one deduces easily that for any $u \in L^{1}(\Omega)$,

$$
\partial J(u)=\left\{p \in K: \int_{\Omega} p(x) u(x) \mathrm{d} x=J(u)\right\} .
$$

We can now give the proof of Proposition 2.2 .

Proof of Proposition 2.2. The Euler equation for problem (3) is

$$
\frac{w-d_{E}}{h}+\partial J(w) \ni 0 .
$$

Set $p=\left(w-d_{E}\right) / h \in-\partial J(w)$. Let also $M=\operatorname{diam} \Omega$. Since $\left|d_{E}\right| \leqslant M$, from the maximum principle also $|w| \leqslant M$ a.e. in $\Omega$. For every $s \in[-M, M]$, let $F_{s}:=\{w<s\}$ (so that in particular $\left.T_{h}(E)=F_{0}\right)$. Since $J(w)<+\infty, F_{s}$ has finite perimeter for almost every $s$.

One has $J(w)=-\int_{\Omega} p w$. By the coarea formula,

$$
J(w)=\int_{-M}^{M} J\left(F_{S}\right) \mathrm{d} s
$$

On the other hand, since $w(x)=M-\int_{w(x)}^{M} \mathrm{~d} s=M-\int_{-M}^{M} \mathbf{1}_{F_{s}}(x) \mathrm{d} s$ for every $x$, one has

$$
-\int_{\Omega} p(x) w(x) \mathrm{d} x=\int_{\Omega} \int_{-M}^{M} p(x) \mathbf{1}_{F_{s}}(x) \mathrm{d} s \mathrm{~d} x-M \int_{\Omega} p(x) \mathrm{d} x .
$$

One easily shows that $\int_{\Omega} p=0$ (since $\left.p \in K\right)$, so that

$$
\int_{-M}^{M} J\left(F_{S}\right) \mathrm{d} s=\int_{-M}^{M} \int_{\Omega} p(x) \mathbf{1}_{F_{S}}(x) \mathrm{d} x \mathrm{~d} s .
$$

But since $p \in K, \int_{\Omega} p \mathbf{1}_{F_{s}} \leqslant J\left(F_{s}\right)$ for every $s$. It follows that for almost every $s, J\left(F_{S}\right)=\int_{\Omega} p \mathbf{1}_{F_{s}}$, that is, $p \in \partial J\left(\mathbf{1}_{F_{s}}\right)$. Let now

$$
I=\left\{s \in[-M, M]: J\left(F_{s}\right)<+\infty \text { and } p \in \partial J\left(\mathbf{1}_{F_{s}}\right)\right\} .
$$


Then $|[-M, M] \backslash I|=0$. Let $s \in I$, and $F \subset \Omega$ have finite perimeter. One has

$$
\begin{aligned}
J(F) & \geqslant J\left(F_{S}\right)+\int_{\Omega}\left(\mathbf{1}_{F}-\mathbf{1}_{F_{s}}\right) p=J\left(F_{S}\right)+\int_{\Omega}\left(\mathbf{1}_{F}-\mathbf{1}_{F_{s}}\right) \frac{w-d_{E}}{h} \\
& =J\left(F_{S}\right)+\int_{\Omega}\left(\mathbf{1}_{F}-\mathbf{1}_{F_{s}}\right) \frac{s-d_{E}}{h}+\int_{\Omega}\left(\mathbf{1}_{F}-\mathbf{1}_{F_{s}}\right) \frac{w-s}{h} .
\end{aligned}
$$

Now, since $F_{s}=\{w<s\}$, we have $\int_{\Omega}\left(\mathbf{1}_{F}-\mathbf{1}_{F_{s}}\right)(w-s) \geqslant 0$. We deduce that

$$
J(F)-\int_{\Omega} \mathbf{1}_{F} \frac{w-s}{h} \geqslant J\left(F_{S}\right)-\int_{\Omega} \mathbf{1}_{F_{s}} \frac{w-s}{h},
$$

that is, $F_{s}$ is a solution to the problem

$$
\min _{F} J(F)+\frac{1}{h} \int_{F}\left(d_{E}(x)-s\right) \mathrm{d} x .
$$

If we now let $E_{s}:=\left\{d_{E}<s\right\}$, then for all $F$ we have

$$
\int_{E_{s} \Delta F}\left|d_{E}-s\right|=\int_{F}\left(d_{E}-s\right)-\int_{E_{s}}\left(d_{E}-s\right) .
$$

Since $\int_{E_{s}}\left(d_{E}-s\right)$ does not depend on $F$, we deduce that $F_{S}$ is also a solution of

$$
\min _{F} J(F)+\frac{1}{h} \int_{F \triangle E_{s}}\left|d_{E}(x)-s\right| \mathrm{d} x .
$$

In particular, observe that $J\left(F_{S}\right) \leqslant 2 M|\Omega| / h$.

If $0 \in I$ we are done. Indeed, the problem that $F_{0}=T_{h}(E)$ solves is the same as $(5)$, although the integral is taken on $F \triangle E_{0}$, instead of $F \triangle E$ in (5); but the difference between these two sets is $E_{0} \backslash E$, on which $d_{E} \equiv 0$. Let us show that $0 \in I$. Observe first that

$$
F_{0}=\{w<0\}=\bigcup_{s<0}\{w<s\}=\bigcup_{s \in I, s<0} F_{s} .
$$

In particular, if $\left(s_{k}\right)_{k} \geqslant 1$ is an increasing sequence of negative numbers in $I$ that converge to 0 , then $\mathbf{1}_{F_{s_{k}}} \rightarrow \mathbf{1}_{F_{0}}$ in $L^{1}(\Omega)$ as $k \rightarrow \infty$. We deduce that $F_{0}$ has finite perimeter, since $J\left(F_{0}\right) \leqslant$ $\liminf _{k \rightarrow \infty} J\left(F_{s_{k}}\right) \leqslant 2 M|\Omega| / h<+\infty$. Moreover, $\int_{\Omega} p \mathbf{1}_{F_{s_{k}}} \rightarrow \int_{\Omega} p \mathbf{1}_{F_{0}} \leqslant J\left(F_{0}\right)$ as $k \rightarrow \infty$. Since $\int_{\Omega} p \mathbf{1}_{F_{s_{k}}}=J\left(F_{s_{k}}\right)$, we deduce that $\int_{\Omega} p \mathbf{1}_{F_{0}}=J\left(F_{0}\right)$. Therefore $p \in \partial J\left(\mathbf{1}_{F_{0}}\right)$, that is, $0 \in I$. (In fact, one shows easily in the same way that $I=[-M, M]$.)

Hence $F_{0}=T_{h}(E)$ is a solution of (5) and the proof of the proposition is complete.

\subsection{The algorithm converges to the generalized Mean Curvature Motion}

Everything that follows comes from the two essential properties that have been shown in Lemma 2.1 and Proposition 2.2. The key is that $T_{h}$ provides a monotone implementation of Almgren, Taylor and Wang's algorithm. We recall Almgren, Taylor and Wang's results first on the set $T_{h}(E)$, then on the evolution $E^{h}(t)$. Notice that the original functional of Almgren, Taylor and Wang is not defined on 
an open bounded set $\Omega$ but on all of $\mathbb{R}^{N}$. Hence the problems will be really equivalent only when $\bar{E} \subset \Omega$ and when the minimizer of $(5)$ is also strictly inside $\Omega$.

As soon as $T_{h}(E)$ lies strictly inside $\Omega$, its perimeter $\mathcal{H}^{N-1}\left(\partial_{*} T_{h}(E)\right)$ coincides with $J\left(T_{h}(E) ; \Omega\right)$ and is independent of $\Omega$ (otherwise, $J\left(T_{h}(E) ; \Omega\right)=\mathcal{H}^{N-1}\left(\partial_{*} T_{h}(E) \cap \Omega\right)$; here $\partial_{*} X$ denotes the measure-theoretic boundary of the set $X$, that is, the complement of the set of points where $X$ has Lebesgue density 1 or 0$)$. Then $T_{h}(E)$ is the minimizer of (5) among all other competitors $F \subset \mathbb{R}^{N}$. In fact, Remark A.3 in Appendix A shows that in this situation, the set $T_{h}(E)=\{w<0\}$ for a solution $w$ of $(3)$ is "independent of $\Omega$ " and would be identical if computed in any larger set $\Omega^{\prime} \supseteq \Omega$.

In particular, if $\overline{\mathrm{co}} E \subset \subset \Omega$, and if one can establish that $T_{h}(\overline{\mathrm{co}} E) \subset \subset \Omega$ for $h$ small enough, then, using [2, 3.1.9], one deduces that for such an $h, T_{h}(\overline{\mathrm{co}} E) \subset \overline{\mathrm{co}} E$. This allows us to conclude that $T_{h}^{n}(E) \subset \overline{\mathrm{co}} E \subset \subset \Omega$ for every $n \geqslant 0$.

In what follows, we will assume for simplicity that $\Omega$ is either a hypercube (or rectangle) or the ball $B(0, R)$ in $\mathbb{R}^{N}$. In the latter case, as soon as $\delta=\operatorname{dist}(E, \partial \Omega)>0$, one shows that also $\operatorname{dist}\left(T_{h}(E), \partial \Omega\right) \geqslant \delta$ when $h$ is small enough (of the order of $\delta^{2}$ ). This is easily shown by comparison (using Lemma 2.1) with the set $T_{h}(B(0, M-\delta)$ ), which can be explicitly computed. If $\Omega$ is a hypercube, the same property is true by Corollary A.7 and Remark A.8

REMARK 2.3 In fact, we conjecture that as soon as $\overline{c o} E \subset \Omega$, then $T_{h}(E) \subset$ co $E$ for $h$ small enough — so that all the results that follow should hold in any open set $\Omega$, as soon as it contains the closed convex envelope of the initial set.

For such $\Omega$, the following results (shown first in [2] by Almgren, Taylor and Wang, but we quote here the statements of Ambrosio [3]) are true.

Theorem 1 ([3, Cor. 3.6, Teo. p. 228], [2, 3.4-3.7]) Assume $E \subset \subset \Omega$ and $T_{h}(E) \subset \subset \Omega$. Then the set $T_{h}(E)$ coincides a.e. with an open set and $\mathcal{H}^{N-1}\left(\partial T_{h}(E) \backslash \partial_{*} T_{h}(E)\right)=0$. Moreover, there exists a closed subset $C$ of $\partial T_{h}(E)$ of dimension less than or equal to $N-8$ (hence empty if $N<8$ ) such that $\partial T_{h}(E) \backslash C$ is a $C^{2, \alpha}$ hypersurface for some $\alpha>0$.

Notice that Corollary A.9 in Appendix A implies that $w$ is Lipschitz in a neighbourhood of $T_{h}(E)$, showing again that $T_{h}(E)=\{w<0\}$ is open. (In fact, the statements of the theorem also hold true for $\partial_{*} T_{h}(E) \cap \Omega$ even when $T_{h}(E)$ is not strictly inside $\Omega$, since the proofs rely on local arguments that are also true in the neighbourhood of each $x \in \partial_{*} T_{h}(E) \cap \Omega$.)

Theorem 2 ([3, pp. 230-232], [2, Thm. 4.4]) Assume $E_{0} \subset \subset \Omega$ (hence $\overline{c o} E_{0} \subset \subset \Omega$ since our choice of $\Omega$ is convex), $\overline{\text { co }} E_{0}$ has diameter $D$ and $\left|\partial E_{0}\right|=0$. There exists $\Gamma$, depending only on $N$, such that for every $t>s \geqslant 0,|t-s| \leqslant 1$,

$$
\left|E^{h}(s) \triangle E^{h}(t)\right| \leqslant(\Gamma+D) J\left(E_{0}\right)\left(\left[\frac{t}{h}\right] h-\left[\frac{s}{h}\right] h\right)^{1 /(n+1)} .
$$

In particular, there exists $E \subset \Omega \times \mathbb{R}_{+}$and a subsequence $\left(E^{h_{k}}\right)_{k} \geqslant 1$ such that for any $T>0$, $E^{h_{k}} \rightarrow E$ in $L^{1}(\Omega \times(0, T)$ ) (in the sense of convergence of the indicator functions), and $E$ satisfies $E(0)=E_{0}$ and

$$
|E(s) \triangle E(t)| \leqslant(\Gamma+D) J\left(E_{0}\right)|t-s|^{1 /(n+1)}
$$

for every $t>s \geqslant 0,|t-s| \leqslant 1$. 
Here we view the sets $E^{h}, E$ as subsets of $\Omega \times \mathbb{R}$, with $E^{h}=\bigcup_{t \in \mathbb{R}} E^{h}(t) \times\{t\}$ while $E(t)$ denotes the section $\{x \in \Omega:(x, t) \in E\}$. More precisely, the convergence of $E^{h_{k}}$ to $E$ is in $L^{\infty}\left(0, T ; L^{1}(\Omega)\right)$.

The following fundamental consistency result is shown by Almgren, Taylor and Wang:

Theorem 3 ([2, Thm. 7.4]) Suppose that $L(t)$ is such that $L(t) \subset \subset \Omega$ for all $0 \leqslant t<t_{0}$ and $\partial L(t)$ is a smooth hypersurface evolving by mean curvature on the interval $\left[0, t_{0}\right)$. Let $E_{0}=L(0)$, $E^{h}(t)$ be defined by (4). Then $E^{h} \rightarrow L$ in $L^{1}\left(\Omega \times\left(0, t_{0}\right)\right)$ as $h \downarrow 0$.

REMARK 2.4 In fact, the proof of Almgren, Taylor and Wang shows that $\partial E^{h}$ goes to $\partial L$ in the Hausdorff distance, in $\Omega \times[0, t]$ for all $t<t_{0}\left(\right.$ and $\left.E^{h} \rightarrow \bar{L},\left(E^{h}\right)^{c} \rightarrow(\stackrel{L}{L})^{c}\right)$.

A consequence of all these results is the following.

THEOREM 4 Assume $E_{0} \subset \subset \Omega$ satisfies $\left|\partial E_{0}\right|=0$ and is such that the viscosity solution $v$ of (1) starting from $\mathbf{1}_{E_{0}^{c}}-\mathbf{1}_{E_{0}}$ is unique [6, 8]. Let $E^{h}$ be defined by 4 . Then $E^{h} \rightarrow E$ in $L^{1}(\Omega \times[0, T])$ for every $T>0$, with $v(x, t)=\mathbf{1}_{E(t) c}(x)-\mathbf{1}_{E(t)}(x)$.

Proof. The proof of this result is a "straightforward" application of the results of Bellettini and Novaga [13], once the properties in Lemma 2.1 and Theorem 3 hold. We fix $T>0$, and choose a subsequence of $\left(E^{h}\right)_{h>0}$ (that we still denote by $\left(E^{h}\right)$ ) such that the Hausdorff limits of $E^{h}$ and of $\left(E^{h}\right)^{c}$ both exist in $\Omega \times[0, T]$. We let $E^{*}=\lim _{h \downarrow 0} E^{h}$ while $E_{*}^{c}=\lim _{h \downarrow 0}\left(E^{h}\right)^{c}$. We can also assume (by Theorem 2 that $E^{h}$ converges to some $E$ in $L^{1}(\Omega \times[0, T])$. Then $E_{*} \subset E \subset E^{*}$, the first one being an open set while the last one is closed. We will show (in Lemma 2.6) that $E_{*}$ is a "barrier" in the sense of Bellettini and Novaga [13, Defs. 2.1 and 2.5] for the mean curvature evolution equation. Next, let $u^{*}=\mathbf{1}_{E_{*}^{c}}-\mathbf{1}_{E_{*}}(x, t)$ for $0 \leqslant t \leqslant T$ and $x \in \mathbb{R}^{N}$ ( $u^{*}$ is naturally extended by 1 outside $\Omega$ ). The function $u^{*}$ is u.s.c. (since $E_{*}$ is open), and for all $\lambda \in \mathbb{R}$, the sets $\left\{u^{*}(\cdot, t)<\lambda\right\}$ are either $\mathbb{R}^{N}, E_{*}(t)$ or $\emptyset$, so that they are always a "barrier". Hence, by [13, Thm. 5.1 $1, u^{*}$ is a viscosity subsolution of 11 in $\Omega \times(0, T)$. In the same way, we deduce that $u_{*}=\mathbf{1}_{\left(E^{*}\right)^{c}}-\mathbf{1}_{E^{*}}$, which is 1.s.c., is a viscosity supersolution of $(1)$.

We denote by $v=\mathbf{1}_{F^{c}}-\mathbf{1}_{F}$ the unique viscosity solution of 1 ip starting from $\mathbf{1}_{E_{0}^{c}}-\mathbf{1}_{E_{0}}$. The assumption that this solution $v$ is unique yields (by [8, Thm. 1.3 and the proof of Thm. 2.1]) that $v^{*}$ (the u.s.c. envelope of $v$ ) is the maximal subsolution of $(1)$ (with initial data $\mathbf{1}_{E_{0}^{c}}-\mathbf{1}_{E_{0}}$ ), while $v_{*}$ (the 1.s.c. envelope of $v$ ) is the minimal supersolution. Hence, if we show that $u^{*}(\cdot, 0) \leqslant \mathbf{1}_{\left(\stackrel{\circ}{E}_{0}\right)^{c}}-\mathbf{1}_{\dot{E}_{0}}=$ $v^{*}(\cdot, 0)$ and $u_{*}(\cdot, 0) \geqslant \mathbf{1}_{\left(\bar{E}_{0}\right)^{c}}-\mathbf{1}_{\bar{E}_{0}}=v_{*}(\cdot, 0)$, we will deduce that $v_{*} \leqslant u_{*} \leqslant u^{*} \leqslant v^{*}$. In other words, $\bar{F} \subseteq E^{*} \supseteq E_{*} \supseteq \stackrel{\circ}{F}$. But the uniqueness of $v$ also yields $|\bar{F} \backslash \stackrel{\circ}{F}|=0$, hence $F=E$ (up to a negligible set).

REMARK 2.5 The uniqueness of the limit evolution $E(t)$ shows that the whole family $\left(E^{h}\right)_{h>0}$ converges (in $L^{1}(\Omega \times[0,+\infty))$ ) to $F$ (whereas $E^{h}$ goes to $\bar{F}$ in the Hausdorff sense in $\Omega \times[0,+\infty$ ), and $\left(E^{h}\right)^{c}$ goes to $(\stackrel{\circ}{F})^{c}$.)

The proof of Theorem 4 will thus be complete if we show the following two assertions:

- $E_{*}$ and $\left(E^{*}\right)^{c}$ are "barriers" in the sense of [13] for the mean curvature evolution (see in particular [13, Defs. 2.1 and 2.5, and the proof of Prop. 2.2]), so that $u^{*}$ and $u_{*}$ are respectively viscosity sub- and supersolutions of equation 11 ;

1 In this particular case Proposition 5.2 of [13] also yields the conclusion. 
- $u^{*}$ and $u_{*}$ are viscosity sub- and supersolutions of (1) with initial data $\mathbf{1}_{E_{0}^{c}}-\mathbf{1}_{E_{0}}$. In the theory of discontinuous viscosity solutions, this is expressed by the fact that $u^{*}$ has to be not greater than the upper semicontinuous envelope of $\mathbf{1}_{E_{0}^{c}}-\mathbf{1}_{E_{0}}$, whereas $u_{*}$ must be not less than its lower semicontinuous envelope.

The first assertion will be consequence of the next Lemma 2.6, whereas the second one is proved in Lemma 2.7

LEMMA 2.6 Let $t_{1}>t_{0} \geqslant 0$ and $\partial L(t)$ be a smooth evolution at a speed larger than the mean curvature on $\left[t_{0}, t_{1}\right]$, in the sense of [13, Def. 2.5]: $L(t)$ is compact for any $t \in\left[t_{0}, t_{1}\right]$, there exists an open set $A \subset \mathbb{R}^{N}$ such that $(t, x) \mapsto d_{L(t)}(x) \in C^{\infty}\left(\left[t_{0}, t_{1}\right] \times A\right), \partial L(t) \subset A$ for any $t$ and

$$
\frac{\partial d_{L}}{\partial t}(t, x)>\Delta d_{L}(t, x)
$$

for any $t \in\left[t_{0}, t_{1}\right]$ and $x \in \partial L(t)$. Then $L\left(t_{1}\right) \subset E_{*}\left(t_{1}\right)$ if $L\left(t_{0}\right) \subset E_{*}\left(t_{0}\right)$, whereas $L\left(t_{1}\right) \subset E^{*}\left(t_{1}\right)^{c}$ if $L\left(t_{0}\right) \subset E^{*}\left(t_{0}\right)^{c}$.

Proof. First of all, by [2] Thm. 7.1], there exists a duration time $\tau>0$ such that for any $t \in\left[t_{0}, t_{1}\right]$, a smooth evolution by mean curvature $F_{t}(s)$ starting from $L(t)$ exists for $t \leqslant s<t+\tau$. One has $F_{t}(t)=L(t)$ and one can check that $\operatorname{dist}\left(\partial F_{t}(s), \partial L(s)\right)$ is (strictly) increasing, so that $L(s) \subset \subset$ $F_{t}(s)$ for each $s \in(t, t+\tau)$. Then [2, Thm. 7.4] shows that if for each $t \in\left[t_{0}, t_{1}\right], s \geqslant t$, and $h>0$, one defines $F_{t}^{h}(s)=T_{h}^{[s / h]-[t / h]} L(t)$, then for $s \in[t, t+\tau)$ the evolution $F_{t}^{h}(s)$ converges to $F_{t}(s)$ as $h \rightarrow 0$, with (in particular) convergence of $\partial F_{t}^{h}(s)$ to $\partial F_{t}(s)$ in the Hausdorff sense (cf. Theorem 3 and Remark 2.4.

Assume $L\left(t_{0}\right) \subset E_{*}\left(t_{0}\right)$. Let us show that for $t \geqslant t_{0}$, if $L(t) \subset E_{*}(t)$, then $L(s) \subset E_{*}(s)$ for every $s \in[t, t+\tau), s \leqslant t_{1}$. This will clearly imply $L\left(t_{1}\right) \subset E_{*}\left(t_{1}\right)$. If $L(t) \subset E_{*}(t)$, then for $h$ small enough, $L(t) \times\{t\}=\{(x, t): x \in L(t)\} \subset \subset E^{h}$. By Lemma 2.1, this shows that if $h$ is small enough, then $F_{t}^{h}(s)=T_{h}^{[s / h]-[t / h]} L(t) \subset E^{h}(s)$ for each $s \geqslant t$, that is, $E^{h}(s)^{c} \subset F_{t}^{h}(s)^{c}$. Since $F_{t}^{h}(s)^{c}$ converges in the Hausdorff sense to $\overline{F_{t}(s)^{c}}$ for each $s \in[t, t+\tau)$, it follows that $E_{*}(s)^{c} \subset \overline{F_{t}(s)^{c}}$ for $t<s<t+\tau$, hence $\dot{\circ}_{t}(s) \subset E_{*}(s)$. But $L(s) \subset \subset F_{t}(s)$, so that $L(s) \subset E_{*}(s)$. This shows the first part of the lemma. The proof of the symmetric statement is similar.

LEMMA $2.7 u^{*}(\cdot, 0) \leqslant \mathbf{1}_{\left(\stackrel{\circ}{E}_{0}\right)^{c}}-\mathbf{1}_{\stackrel{E}{0}_{0}}$ and $u_{*}(\cdot, 0) \geqslant \mathbf{1}_{\left(\bar{E}_{0}\right)^{c}}-\mathbf{1}_{\bar{E}_{0}}$.

Proof. We only prove the first assertion. It is enough to show that if $x \in \stackrel{\circ}{E}_{0}$, then $u^{*}(x, 0)=-1$, that is, $x \in E_{*}(0)$. Let $\varepsilon>0$ be such that $B=B(x, \varepsilon) \subset \subset \stackrel{\circ}{E}_{0}$. We deduce that $T_{h}^{[t / h]}(B) \subset E^{h}(t)$ for every $t$. By arguments similar to those invoked in the proof of the previous lemma, we find that $\left\{(y, t):|y-x|^{2}<\varepsilon^{2}-2(N-1) t\right\} \subset E_{*}$, so that $x \in E_{*}(0)$. The proof of Theorem 4 is now complete.

\section{Implementation}

In order to implement the algorithm, one needs to implement successively

- the computation of the (signed) distance function to the level set 0 of a function $w$;

- the resolution of problem (3). 
We will describe these two steps in dimension 2. The first step is done by using an implementation of the fast marching algorithm, introduced by Tsitsiklis in [56]. We follow [52]. The second step is done using the fixed-point dual algorithm of [21].

\subsection{The fast marching algorithm}

Our implementation of the fast marching algorithm is the following. The idea is to solve the eikonal equation $|\nabla d|=1$ inside and outside the set $E$, with Dirichlet data $d=0$ on $\partial E$.

The input of the algorithm is an array $w_{i, j}$ of values that corresponds to the discretization of a function $w(x)$ defined on the open domain $\Omega \subset \mathbb{R}^{2}$ and such that $E=\{w<0\}$. To simplify, we assume $\Omega$ is a rectangle, so that the array $w_{i, j}$ is defined for $1 \leqslant i \leqslant N, 1 \leqslant j \leqslant M$. We also assume that the discretization step is 1 .

The output of the algorithm must be the values $d_{i, j}$ of the signed distance function to $\partial E$.

In a first step, we compute a nonnegative distance function (that is, the actual distance to $\partial E$ ). In what follows we still denote it by $d_{i, j}$. The principle of the fast marching algorithm is to compute the distance $d_{i, j}$ starting from the points with smallest distance, and, at each step, to try to find the position $(i, j)$ such that the distance $d_{i, j}$ should be the smallest among all points where it has not been computed yet (the actual implementation in [56] is a bit more precise, we refer to that paper for details). In order to do that we need to keep track of the order (determined by the distance $d_{i, j}$ ) of all the points that have already been processed: one way to do that is to maintain for each $(i, j)$ a pointer next $(i, j)$ to the position $\left(i^{\prime}, j^{\prime}\right)$ such that $d_{i^{\prime}, j^{\prime}}$ is minimal among all other points already calculated, and where the distance is larger than $d_{i, j}$. This array next(.) has to be updated each time a new value of $d$ is calculated.

We introduce a threshold $S$ which is the maximum distance (in absolute value) that we want to compute. We have observed experimentally that this does not change the output as long as $S$ is larger than the distance between the original curve $\partial E$ and the evolved curve $\partial T_{h}(E)$ that will be computed. If the curvature is very large, then $S$ has to be increased. It can be chosen initially larger than $\operatorname{diam}(\Omega)$ so that no thresholding is done.

Initialization step. In the initialization step, we let first $d_{i, j}=S$ for all $i, j$. A convenient way to deal with the boundary $\partial \Omega$ is to let also $d_{i, j}>S$ if $(i, j)$ is at the boundary, that is, whenever $i \in\{1, N\}$ or $j \in\{1, M\}$. Moreover, one decides never to change the points where $d_{i, j}>S$. In this way, no other precaution needs to be taken. On the other hand, the distance will not be computed at these points, which is a minor drawback. Another possibility is to consider periodic boundary conditions.

Thus, for each $(i, j)$, one considers the following three (mutually exclusive) situations:

(i) $w_{i, j}=0$ (this in fact should almost never occur...), in which case we let $d_{i, j}=0$.

(ii) $w_{i, j}>0$ : then we check whether one (or several) of the neighbouring points $i \pm 1, j, i, j \pm 1$ have nonpositive $w$. In this case, the point is near $\partial E$. We evaluate the distance of $(i, j)$ to $\partial E$. If $w_{i, j \pm 1}>0$ then $\partial E$ crosses only one of the segments $[(i-1, j),(i, j)]$ and $[(i, j),(i+1, j)]$ (or both). If $w_{i-1, j} \leqslant 0$, we assume that $\partial E$ crosses $[(i-1, j),(i, j)]$ at $\left(i-w_{i, j} /\left(w_{i, j}-w_{i-1, j}\right), j\right)$, hence at distance $d_{i, j}=w_{i, j} /\left(w_{i, j}-w_{i-1, j}\right)$. If $w_{i+1, j} \leqslant 0$, then $\partial E$ crosses $[(i, j),(i+1, j)]$ at $\left(i+w_{i, j} /\left(w_{i, j}-w_{i+1, j}\right), j\right)$, that is, at distance $d_{i, j}=w_{i, j} /\left(w_{i, j}-w_{i+1, j}\right)$. If both $w_{i-1, j} \leqslant 0$ and $w_{i+1, j} \leqslant 0$, then $d_{i, j}$ is taken to be the minimum of the previous two values. 
On the other hand, if $w_{i \pm 1, j}>0$, then $\partial E$ crosses the segments $[(i, j-1),(i, j)]$ or/and $[(i, j),(i, j+1)]$, and we compute the distance $d_{i, j}$ in the same way.

The last situation is when $\partial E$ crosses both the horizontal and vertical axes near $(i, j)$. Assume for instance $w_{i+1, j} \leqslant 0$ and $w_{i, j+1} \leqslant 0$. Then the distance $d$ is zero at the points $\left(i+w_{i, j} /\left(w_{i, j}-w_{i+1, j}\right), j\right)$ and $\left(i, j+w_{i, j} /\left(w_{i, j}-w_{i, j+1}\right)\right)$. In other words, the gradient of $d$ at $(i, j)$ is approximated by $d_{i, j} \times\left(1-w_{i+1, j} / w_{i, j}, 1-w_{i, j+1} / w_{i, j}\right)$. The equation $|\nabla d|=1$ gives the value of $d_{i, j}: d_{i, j}=1 /\left|\left(1-w_{i+1, j} / w_{i, j}, 1-w_{i, j+1} / w_{i, j}\right)\right|$. If $\partial E$ crosses more than one horizontal or vertical axis near $(i, j)$, then we take for $d_{i, j}$ the minimum of all possible values.

(iii) $w_{i, j}<0$ : then we check whether $w_{i \pm 1, j}$ or $w_{i, j \pm 1} \geqslant 0$. If so, we compute the distance $d_{i, j}$ as in the previous case.

If the distance has been computed at point $(i, j)$, then we update the variables "next". We first find the position of $(i, j)$ in the array, by finding the first $\left(i^{\prime}, j^{\prime}\right)$ for which $d_{\text {next }\left(i^{\prime}, j^{\prime}\right)} \geqslant d_{i, j}$. We then set next $(i, j)=\operatorname{next}\left(i^{\prime}, j^{\prime}\right)$ and $\operatorname{next}\left(i^{\prime}, j^{\prime}\right)=(i, j)$.

We iterate this until all points with $d_{i, j}=S$ are not near $\partial E$ (we assume $S$ has not been chosen too small!, so that points in the neighbourhood of $\partial E$ are all at distance less than $S$ ).

Main iteration. Once the distance function has been calculated near $\partial E$, the "fast marching" process can begin. For every point $(i, j)$ that has already been calculated (that is, where $d_{i, j}<S$ ), starting from the first (with least distance) and following the pointer "next", we check the 4 neighbours $(i \pm 1, j)$ and $(i, j \pm 1)$ of $(i, j)$. If at one of these points, the distance has not been calculated yet, we compute it. In order to compute the distance at some neighbour $d_{i^{\prime}, j^{\prime}}$, we use the standard discretization of the eikonal equation (see [50]):

$$
\left|\left(\max \left\{d_{i^{\prime}, j^{\prime}}-d_{i^{\prime}-1, j^{\prime}}, d_{i^{\prime}, j^{\prime}}-d_{i^{\prime}+1, j^{\prime}}, 0\right\}, \max \left\{d_{i^{\prime}, j^{\prime}}-d_{i^{\prime}, j^{\prime}-1}, d_{i^{\prime}, j^{\prime}}-d_{i^{\prime}, j^{\prime}+1}, 0\right\}\right)\right|=1 .
$$

If the distance has been updated at some point, we also update the array next accordingly. If the new distance was higher than $S$ we truncate it to $S$. The loop stops when it is impossible to compute a new distance which is less than $S$ (or when all points $(i, j)$ have been updated).

In Section 4.3 we explain how to adapt the method to compute anisotropic distances.

Post-treatment. Once the unsigned distance function to $\partial E$ has been calculated, it remains to change $d_{i, j}$ to $-d_{i, j}$ at each position $(i, j)$ where $w_{i, j}<0$.

\subsection{A numerical algorithm for the minimization of the total variation}

In order to compute the minimizer of (3), we use the algorithm proposed in [21]. Following the notations of that paper, we let $X=\mathbb{R}^{N \times M}, Y=X \times X$, and we define the gradient operator $\nabla: X \rightarrow Y$ by

$$
(\nabla u)_{i, j}=\left((\nabla u)_{i, j}^{1},(\nabla u)_{i, j}^{2}\right)
$$

with

$$
\begin{aligned}
& (\nabla u)_{i, j}^{1}= \begin{cases}u_{i+1, j}-u_{i, j} & \text { if } i<N, \\
0 & \text { if } i=N,\end{cases} \\
& (\nabla u)_{i, j}^{2}= \begin{cases}u_{i, j+1}-u_{i, j} & \text { if } j<M, \\
0 & \text { if } j=M,\end{cases}
\end{aligned}
$$


for $i=1, \ldots, N, j=1, \ldots, M$. We define the discrete total variation as

$$
J^{d}(u)=\sum_{i=1}^{N} \sum_{j=1}^{M}\left|(\nabla u)_{i, j}\right|
$$

In $X$ and $Y$ we consider the standard scalar products, that is,

$$
\left\langle x, x^{\prime}\right\rangle=\sum_{i=1}^{N} \sum_{j=1}^{M} x_{i, j} x_{i, j}^{\prime}
$$

for any $x, x^{\prime} \in X$, and

$$
\left\langle y, y^{\prime}\right\rangle=\sum_{i=1}^{N} \sum_{j=1}^{M} y_{i, j} \cdot y_{i, j}^{\prime}=\sum_{i=1}^{N} \sum_{j=1}^{M}\left(y_{i, j}^{1} y_{i, j}^{\prime 1}+y_{i, j}^{2} y_{i, j}^{\prime 2}\right)
$$

for any $y, y^{\prime} \in Y$. In order to find a definition of $J^{d}$ similar to (2), we introduce the operator $\operatorname{div}: Y \rightarrow X$, defined by $\operatorname{div}=-\nabla^{*}$, that is, for $\xi=\left(\xi^{1}, \xi^{2}\right) \in Y$,

$$
(\operatorname{div} \xi)_{i j}=\left\{\begin{array}{ll}
\xi_{i, j}^{1}-\xi_{i-1, j}^{1} & \text { if } 1<i<N, \\
\xi_{i, j}^{1} & \text { if } i=1, \\
-\xi_{i-1, j}^{1} & \text { if } i=N,
\end{array}+ \begin{cases}\xi_{i, j}^{2}-\xi_{i, j-1}^{2} & \text { if } 1<j<M, \\
\xi_{i, j}^{2} & \text { if } j=1, \\
-\xi_{i, j-1}^{2} & \text { if } j=M .\end{cases}\right.
$$

It satisfies $\langle\operatorname{div} \xi, u\rangle=-\langle\xi, \nabla u\rangle$ for any $\xi \in Y$ and $u \in X$.

It is then not difficult to show that

$$
J^{d}(u)=\sup \left\{\langle u, \operatorname{div} \xi\rangle:\left|\xi_{i, j}\right| \leqslant 1 \forall i, j\right\} .
$$

The discrete problem now consists in solving

$$
\min _{w \in X} J^{d}(w)+\frac{\|w-d\|^{2}}{2 h},
$$

where $\|x\|=\langle x, x\rangle$ is the Euclidean norm and $d=\left(d_{i, j}\right)$ is the signed distance function evaluated by the algorithm of Section 3.1] In [21] it is shown that the solution $w$ of $(8)$ is given by

$$
w=d-\pi_{h K}(d),
$$

where $\pi_{h K}$ is the orthogonal projection onto the convex set $h K$,

$$
K=\left\{\operatorname{div} \xi:\left|\xi_{i, j}\right| \leqslant 1 \forall i=1, \ldots, N, j=1, \ldots, M\right\}
$$

being the closed convex set such that $J^{d}(u)=\sup _{v \in K}\langle v, u\rangle$. The difficulty is hence to compute this nonlinear projection. One has to solve

$$
\min \left\{\|h \operatorname{div} \xi-d\|^{2}: \xi \in Y,\left|\xi_{i, j}\right| \leqslant 1 \forall i=1, \ldots, N, j=1, \ldots, M\right\} .
$$

Introducing the Lagrange multipliers $\alpha_{i, j}$ associated to the constraint $\left|\xi_{i, j}\right|^{2}-1 \leqslant 0$, we obtain the following Euler equation:

$$
-(\nabla(h \operatorname{div} \xi-d))_{i, j}+\alpha_{i, j} \xi_{i, j}=0
$$


for all $i, j$, with either $\alpha_{i, j}>0$ and $\left|\xi_{i, j}\right|=1$, or $\left|\xi_{i, j}\right|<1$ and $\alpha_{i, j}=0$. In the latter case, also $(\nabla(h \operatorname{div} \xi-d))_{i, j}=0$; hence in any case $\alpha_{i, j}=\left|(\nabla(h \operatorname{div} \xi-d))_{i, j}\right|$. The Euler equation is therefore

$$
-(\nabla(h \operatorname{div} \xi-d))_{i, j}+\left|(\nabla(h \operatorname{div} \xi-d))_{i, j}\right| \xi_{i, j}=0
$$

for all $i, j$. The form of the Euler equation suggests the following iterative method: we let $\xi^{0}=0$, and for each $n=1,2, \ldots$ we let

$$
\xi_{i, j}^{n}=\frac{\xi_{i, j}^{n-1}+\tau\left(\nabla\left(\operatorname{div} \xi^{n-1}-d / h\right)\right)_{i, j}}{1+\tau\left|\left(\nabla\left(\operatorname{div} \xi^{n-1}-d / h\right)\right)_{i, j}\right|},
$$

where $\tau>0$ is a fixed parameter.

It is shown in [21] that as soon as $\tau \leqslant 1 / 8$, the iteration converges, and $d-h \operatorname{div} \xi^{n}$ goes to the solution $w$ of (8) as $n \rightarrow \infty$.

Once this solution has been computed, one goes back to Section 3.1 to evaluate the new signed distance $d$ to the boundary of $\{w<0\}$.

\subsection{Examples}

We just show two examples of evolutions computed with this algorithm. The initial curve is shown on the left, and various steps of the evolution are shown on the right. In both examples the size of the grid is $150 \times 150$.
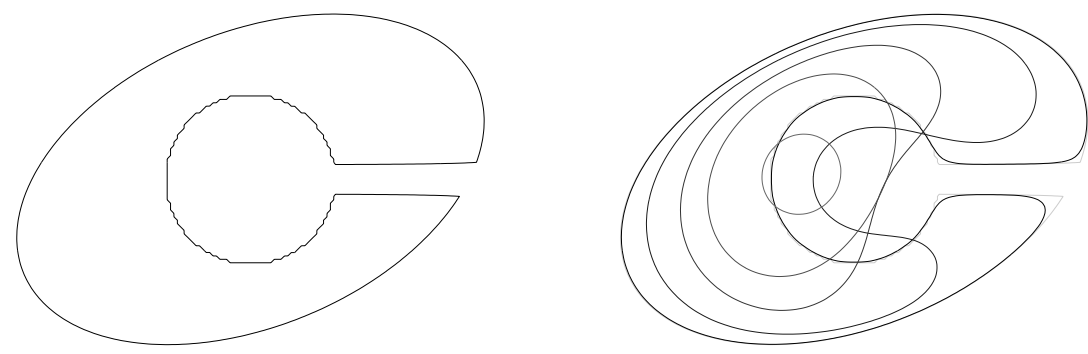

FIG. 1. An original curve (left), and its evolution for times $t=1,30,70,100,140$ (right), as computed by the algorithm.
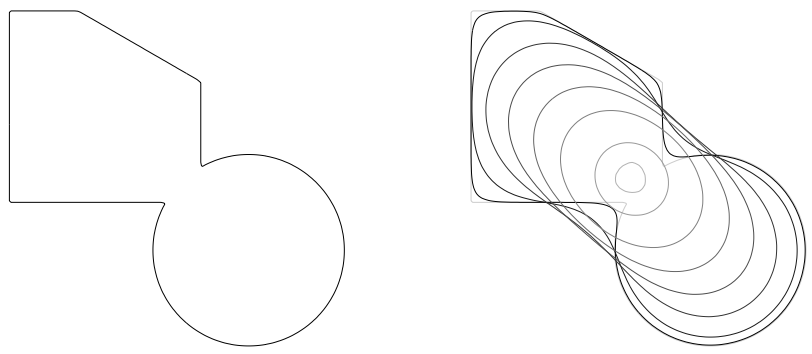

FIG. 2. Another example, showing iterations 1, 10, 30, 50, 70, 90, 110, 116. 


\section{The anisotropic case}

\subsection{Description of the Anisotropic Mean Curvature Motion}

In this section we discuss the anisotropic situation. We first consider anisotropic variants of the total variation $J$. We assume $\varphi$ is a convex, even, 1-homogeneous function, with $a|\xi| \leqslant \varphi(\xi) \leqslant b|\xi|$ for every $\xi \in \mathbb{R}^{N}(a, b>0)$. We define $J_{\varphi}$ by

$$
J_{\varphi}(u ; \Omega)=\sup \left\{\int_{\Omega} u(x) \operatorname{div} \xi(x) \mathrm{d} x: \xi \in C_{c}^{1}\left(\Omega ; \mathbb{R}^{d}\right), \varphi(\xi(x)) \leqslant 1 \forall x \in \Omega\right\} .
$$

It is clear that $J_{\varphi}(u ; \Omega)<+\infty$ iff $u$ has bounded variation. Introducing the polar function $\varphi^{\circ}$ given by

$$
\varphi^{\circ}(\eta)=\sup _{\varphi(\xi) \leqslant 1} \xi \cdot \eta,
$$

one shows that $J_{\varphi}(u ; \Omega)=\varphi^{\circ}(D u)(\Omega)=\int_{\Omega} \varphi^{\circ}(D u /|D u|) \mathrm{d}|D u|$. The anisotropic perimeter of a set $F \subset \Omega$ is $J_{\varphi}(F ; \Omega)=J_{\varphi}\left(\mathbf{1}_{F} ; \Omega\right)=\int_{\partial_{*} F \cap \Omega} \varphi^{\circ}\left(v_{F}\right) \mathrm{d} \mathcal{H}^{N-1}$; the quantity $\varphi^{\circ}\left(v_{F}\right)$ introduces a weight on the surface that depends on its orientation.

Notice that $d_{\varphi}(x, y)=\varphi(x-y)$ is a distance in $\mathbb{R}^{N}$. Given the set $E \subset \Omega$ we introduce the signed distance $d_{E}^{\varphi}$ to $\partial E$ by

$$
d_{E}^{\varphi}(x)=\inf _{y \in E} \varphi(x-y)-\inf _{y \notin E} \varphi(x-y) .
$$

For a definition of the Mean Curvature Motion in the presence of anisotropy we refer to [18]. The anisotropic curvature of $\partial E$ at $x$ is given by $\kappa_{\varphi}=\operatorname{div} \nabla \varphi^{\circ}\left(\nabla d_{E}^{\varphi}\right)$ (or is an element of $\operatorname{div} \partial \varphi^{\circ}\left(\nabla d_{E}^{\varphi}\right)$ whenever $\varphi^{\circ}$ is not smooth). The definition proposed in [18] assumes that the surfaces evolve with velocity $\kappa_{\varphi}$ along the " $\varphi$-normal" $n_{\varphi}=\nabla \varphi^{\circ}\left(\nabla d_{E}^{\varphi}\right)$. The corresponding equation, in the viscosity sense, is

$$
\frac{\partial u}{\partial t}=\varphi^{\circ}(\nabla u) \operatorname{div} \nabla \varphi^{\circ}(\nabla u) \text {. }
$$

In order for the equation to be well defined one needs to assume that both $\varphi$ and $\varphi^{\circ}$ are smooth (away from 0 ). This excludes the so-called crystalline case, in which the boundary "Wulff shape" $\{\varphi \leqslant 1\}$ may have flat parts or angles. Following [2], we will assume that $\varphi^{\circ}$ (which is $\Phi$ in [2]) is $C^{3, \alpha}$ and elliptic (elliptic meaning that for $x \neq 0$ and $e \in \mathbb{R}^{N},\left(D^{2} \varphi^{\circ}(x) e\right) \cdot e$ vanishes only whenever $e \in \mathbb{R} x$ ). This yields a similar smoothness for $\varphi$ (see Remark 4.1 below).

Let us point out that equation (11) slightly differs from what is considered in Almgren, Taylor and Wang's paper [2]. They consider the evolution

$$
\frac{\partial u}{\partial t}=|\nabla u| \operatorname{div} \nabla \varphi^{\circ}(\nabla u)
$$

that corresponds to the evolution with velocity $\kappa_{\varphi}$ along the Euclidean normal vector to the surface. It is not clear to us which point of view is more "natural". In any case, as long as $\varphi^{\circ}$ is smooth and elliptic, the results established in [2] for equation (12) are also valid for equation (11). See also [34]. Almgren, Taylor and Wang consider the evolution given by $E(t)=\lim _{h \rightarrow 0} T_{h}^{[t / h]}\left(E_{0}\right)$ with the operator $T_{h}$ defined by: $F=T_{h}(E)$ is a solution of

$$
\min _{F} J_{\varphi}(F ; \Omega)+\frac{1}{h} \int_{F \triangle E}\left|d_{E}(x)\right| \mathrm{d} x
$$

(in fact for $\Omega=\mathbb{R}^{N}$ ). They use the Euclidean distance in the second term of the functional. Here we chose to consider instead the problem 


$$
\min _{F} J_{\varphi}(F ; \Omega)+\frac{1}{h} \int_{F \triangle E}\left|d_{E}^{\varphi}(x)\right| \mathrm{d} x,
$$

which is introduced in [18, §5.2]. The modification of our algorithm to the case of (13) is obvious. On the other hand, for the second problem, the fact that the evolution of the Wulff shape is selfsimilar is quite obvious also in the crystalline case (since it can be transformed into a basic onedimensional problem exactly as in the Euclidean case). For the study of Almgren, Taylor and Wang's evolution in the crystalline case see [1].

\subsection{The algorithm}

Let us briefly describe our algorithm in the anisotropic case. Instead of 3 , given $E \subset \Omega$, we now solve

$$
\min _{w \in L^{2}(\Omega)} \int_{\Omega} \frac{\left(w(x)-d_{E}^{\varphi}(x)\right)^{2}}{2 h} \mathrm{~d} x+J_{\varphi}(w ; \Omega) .
$$

again, the solution $w$ is unique, and we let $T_{h}(E)=\{w<0\}$.

The evolution starting from a set $E_{0}$ is defined as in Section 2.1. by (4). Then Lemma 2.1 also holds (the algorithm is monotone), as also does the counterpart of Proposition 2.2 that is, $T_{h}(E)$ is a solution of 14.

As long as $\varphi^{\circ}$ is smooth (meaning, as in [2], $C^{3, \alpha}$ off 0 for some $\alpha>0$ ) and elliptic, the other results in Section 2 still hold, including Theorem 4, with $v$ the viscosity solution of (11). The only difference in the proof is that in Lemma 2.6, the inequality (6) must be replaced with the anisotropic version

$$
\frac{\partial d_{L}^{\varphi}}{\partial t}(t, x)>D^{2} \varphi^{\circ}\left(\nabla d_{L}^{\varphi}(t, x)\right): D^{2} d_{L}^{\varphi}(t, x) .
$$

REMARK 4.1 Observe that if $\varphi^{\circ}$ is $C^{k, \alpha}, k \geqslant 1$, and elliptic, then also $\varphi$ is $C^{k, \alpha}$ (off 0 ) and the Wulff shape $\{\varphi \leqslant 1\}$ is smooth and uniformly convex. This can been shown by using the fact that $\varphi(x)^{2} / 2=\left(\varphi^{\circ 2} / 2\right)^{* *}(x)=\max _{y}\left(x \cdot y-\varphi^{\circ}(y)^{2} / 2\right)$. The maximum is reached at $y$ such that $x=\varphi^{\circ}(y) \nabla \varphi^{\circ}(y):=T^{\circ}(y)$, and $y \neq 0$ if $x \neq 0$. The ellipticity implies that $y$ is unique, and by the local inversion theorem, there exists $T=\left(T^{\circ}\right)^{-1}$ which is $C^{1}$ off 0 . Then one shows easily that $T$ is $C^{k-1, \alpha}$, so that $\varphi(x)^{2} / 2=x \cdot T(x)-\varphi^{\circ}(T(x))^{2} / 2$ has the same regularity, and that $T=\varphi \nabla \varphi$, so that $\nabla \varphi$ has the same regularity. The uniform convexity of the Wulff shape follows from the regularity of $\varphi^{\circ}$ (one shows that if it were not uniformly convex, $\nabla \varphi^{\circ}$ would not be continuous).

\subsection{Implementation}

The algorithm is implemented in the same way as in the isotropic case. The computation of the anisotropic distance is just as easy, except for minor modifications that we explain below. The algorithm for minimizing the anisotropic total variation is also implemented in the same way. Unfortunately, the convergence seems very slow in this case, and we could not find any proof of convergence.

4.3.1 The anisotropic distance. In order to compute the anisotropic distance $d_{E}^{\varphi}$ for $E=$ $\{w<0\}$, we adapt the fast-marching algorithm described in Section 3.1 to the computation of the solution of the eikonal equation $\varphi^{\circ}\left(\nabla d_{E}^{\varphi}\right)=1$. Everything is the same except that in the 
initialization step, items (ii) and (iii) have to be modified, as well as equation (7) in the main iteration. For the sake of simplicity, we consider only anisotropies that are even in both directions $\left(\varphi(x, y)=\varphi( \pm x, y)=\varphi(x, \pm y)\right.$ for all $\left.(x, y) \in \mathbb{R}^{2}\right)$.

In item (ii) of the initialization, we proceed almost as in the isotropic case. If $w_{i, j}>0$ and $w_{i-1, j} \leqslant 0$ we assume again that $\partial E$ crosses $[(i-1, j),(i, j)]$ at $\left(i-h^{-}, j\right)$ for $h^{-}=$ $w_{i, j} /\left(w_{i, j}-w_{i-1, j}\right)$, on the other hand, if $w_{i-1, j}>0$, we let $h^{-}=+\infty$. Similarly, we define $h^{+}=w_{i, j} /\left(w_{i, j}-w_{i+1, j}\right)$ if $w_{i+1, j} \leqslant 0, h^{+}=+\infty$ otherwise, and $v^{ \pm}=w_{i, j} /\left(w_{i, j}-w_{i, j \pm 1}\right)$ if $w_{i, j \pm 1} \leqslant 0$, and $+\infty$ otherwise. Then we let $h=\min \left\{h^{+}, h^{-}\right\}$and $v=\min \left\{v^{+}, v^{-}\right\}$, and when at least one of these values is finite, we assume that the gradient of $d^{\varphi}$ at $(i, j)$ is $\left(d_{i, j}^{\varphi} / h, d_{i, j}^{\varphi} / v\right)$. The eikonal equation implies $d_{i, j}^{\varphi}=1 / \varphi^{\circ}(1 / h, 1 / v)$. Item (iii) is treated in the same way.

Then, in the main iteration, we must replace equation (7) with

$$
\varphi^{\circ}\left(\max \left\{d_{i^{\prime}, j^{\prime}}^{\varphi}-d_{i^{\prime}-1, j^{\prime}}^{\varphi}, d_{i^{\prime}, j^{\prime}}^{\varphi}-d_{i^{\prime}+1, j^{\prime}}^{\varphi}, 0\right\}, \max \left\{d_{i^{\prime}, j^{\prime}}^{\varphi}-d_{i^{\prime}, j^{\prime}-1}^{\varphi}, d_{i^{\prime}, j^{\prime}}^{\varphi}-d_{i^{\prime}, j^{\prime}+1}^{\varphi}, 0\right\}\right)=1 .
$$

This means that given four real numbers $a, b, c, d$, we need to be able to solve the equation $\varphi^{\circ}(\max \{x-a, x-b, 0\}, \max \{x-c, x-d, 0\}=1$. If (for instance) $a \leqslant b$ and $c \leqslant d$, this equation clearly reduces to $\varphi^{\circ}\left((x-a)^{+},(x-c)^{+}\right)=1$ (where $t^{+}=\max \{t, 0\}$ denotes the positive part).

If $a=c$, then clearly $x=a+1 / \varphi^{\circ}(1,1)$.

If $a<c$, then $x$ can be between $a$ and $c$, in which case the equation is $\varphi^{\circ}(x-a, 0)=1$ so that $x=a+1 / \varphi^{\circ}(1,0)$. If the latter value is larger than $c$, then $x$ is not between $a$ and $c$, that is, $x>c$, and the equation is $\varphi^{\circ}(x-a, x-c)=1$. Letting $t=x-c, t_{0}=c-a$, we must find $t>0$ solving $\varphi^{\circ}\left(t_{0}+t, t\right)=1$, or $\varphi^{\circ}\left(1+t / t_{0}, t / t_{0}\right)=1 / t_{0}$. This means that we must find some efficient way to invert the function $s \mapsto \varphi^{\circ}(1+s, s), s>0$. There are many ways to do this (which might depend on the particular function $\varphi^{\circ}$ ) and we do not want to discuss this point here.

If $a>c$ the situation is the same. If $x$ is between $a$ and $c$, then $x=c+1 / \varphi^{\circ}(0,1)$, otherwise, if the latter value is greater than $a$, then $x>a>c$ and the equation is $\varphi^{\circ}(x-a, x-c)=1$; this time, if we let $t=x-a$ and $t_{0}=a-c$ we have to find $t>0$ that solves $\varphi^{\circ}\left(t, t_{0}+t\right)=1$, or $\varphi^{\circ}\left(t / t_{0}, 1+t / t_{0}\right)=1 / t_{0}$.

The rest of the algorithm is as in the isotropic case.

4.3.2 Anisotropic total variation minimization. The discrete total variation is, in the anisotropic case,

$$
J_{\varphi}^{d}(u)=\sum_{i=1}^{N} \sum_{j=1}^{M} \varphi^{\circ}\left((\nabla u)_{i, j}\right)
$$

and one has $J_{\varphi}^{d}(u)=\sup _{v \in K_{\varphi}}\langle v, u\rangle$ with $K_{\varphi}$ given by

$$
K_{\varphi}=\left\{\operatorname{div} \xi: \varphi\left(\xi_{i, j}\right) \leqslant 1 \forall i=1, \ldots, N, j=1, \ldots, M\right\} .
$$

Once the distance function $d^{\varphi}$ has been numerically computed by the algorithm in the previous section, the discrete problem consists in solving

$$
\min _{w \in X} J_{\varphi}^{d}(w)+\frac{\left\|w-d^{\varphi}\right\|^{2}}{2 h},
$$

and again the solution is given by

$$
w=d^{\varphi}-\pi_{h K_{\varphi}}\left(d^{\varphi}\right) .
$$


One has to solve (9) with the constraint on the $\xi_{i, j}$ replaced with $\varphi\left(\xi_{i, j}\right) \leqslant 1$ for every $i, j$ (and $d$ replaced with $\left.d^{\varphi}\right)$. We introduce the operators $T$ and $T^{\circ}$ defined by $T=\partial\left(\varphi^{2} / 2\right)=\varphi \partial \varphi$ and $T^{\circ}=\partial\left(\varphi^{\circ 2} / 2\right)=\varphi^{\circ} \partial \varphi^{\circ}$. In the smooth case, the subgradients are in fact gradients and $T(\xi)=$ $\varphi(\xi) \nabla \varphi(\xi)$, etc., but we will perform computation also in the nonsmooth case (with $\varphi$ and/or $\varphi^{\circ}$ just Lipschitz) where $T$ and $T^{\circ}$ can be multivalued. Notice that in any case, the functions $\varphi^{2} / 2$ and $\varphi^{\circ 2} / 2$ are Fenchel conjugates, so that $x \in T(\xi)$ iff $\xi \in T^{\circ}(x)$.

As in the isotropic case, we introduce Lagrange multipliers $\alpha_{i, j} \geqslant 0$ associated to the constraints $\varphi\left(\xi_{i, j}\right)^{2}-1 \leqslant 0$, and find the following Euler equation:

$$
-\left(\nabla\left(h \operatorname{div} \xi-d^{\varphi}\right)\right)_{i, j}+\alpha_{i, j} T\left(\xi_{i, j}\right) \ni 0
$$

for all $i, j$. Here, $\alpha_{i, j}>0$ only when $\varphi\left(\xi_{i, j}\right)=1$, and since in this case $\varphi^{\circ}\left(\alpha_{i, j} T\left(\xi_{i, j}\right)\right)=$ $\alpha_{i, j} \varphi\left(\xi_{i, j}\right) \varphi^{\circ}\left(\partial \varphi\left(\xi_{i, j}\right)\right)=\alpha_{i, j}$, we find that

$$
\alpha_{i, j}=\varphi^{\circ}\left(\left(\nabla\left(h \operatorname{div} \xi-d^{\varphi}\right)\right)_{i, j}\right)
$$

We choose, as in the isotropic case, $\xi^{0}=0$. Then the counterpart of the iterative method of [21], given by the formula 10, , consists in updating $\xi^{n-1}$ by means of the semi-implicit scheme

$$
\xi_{i, j}^{n}=\xi_{i, j}^{n-1}-\tau\left[-\left(\nabla\left(\operatorname{div} \xi^{n-1}-d^{\varphi} / h\right)\right)_{i, j}+\varphi^{\circ}\left(\left(\nabla\left(\operatorname{div} \xi^{n-1}-d^{\varphi} / h\right)\right)_{i, j}\right) T\left(\xi_{i, j}^{n}\right)\right] .
$$

This leads to the iteration

$$
\xi_{i, j}^{n}=\left(I+\tau \varphi^{\circ}\left(\left(\nabla\left(\operatorname{div} \xi^{n-1}-d^{\varphi} / h\right)\right)_{i, j}\right) T\right)^{-1}\left(\xi_{i, j}^{n-1}+\tau\left(\nabla\left(\operatorname{div} \xi^{n-1}-d^{\varphi} / h\right)\right)_{i, j}\right) .
$$

We recall that for any $s \geqslant 0$, the operator $(I+s T)^{-1}$ is singlevalued, so that the iteration $(19)$ is well defined. In fact, for any $\zeta \in \mathbb{R}^{2},(I+s T)^{-1} \zeta$ is the unique minimizer in $\mathbb{R}^{2}$ of $\xi \mapsto(\xi-\zeta)^{2}+s \varphi(\xi)^{2}$.

We have no proof of convergence for this algorithm. In practice, we found that for the same $\tau$ as in the isotropic case $(\tau=1 / 8)$, it seems that $d^{\varphi}-h \operatorname{div} \xi^{n}$ goes to $w$, although quite slowly, as $n \rightarrow \infty$. We performed our computations in the cases where $\{\varphi \leqslant 1\}$ (the Wulff shape) is an equilateral hexagon and a square. In the next section we show some results.

4.3.3 Two examples. We performed numerical calculations with a nonsmooth anisotropy, although the consistency and convergence theorems are not true in this case. However, the examples show that the algorithm computes what is expected to be the correct motion. As pointed out in the
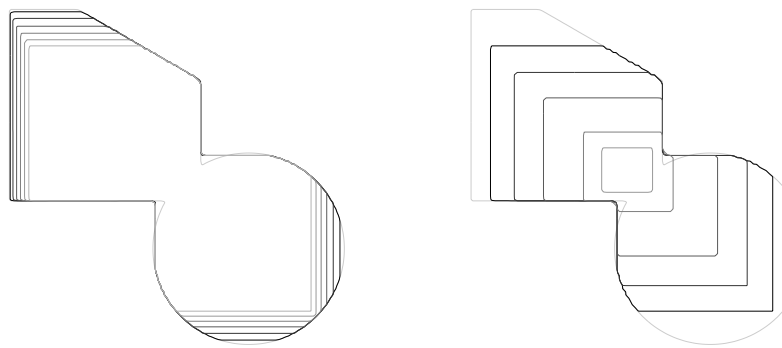

FIG. 3. The evolution at iterations 1, 4, 8, 12, 16, 20 and 20, 40, 60, 80, 88 (square Wulff shape). 

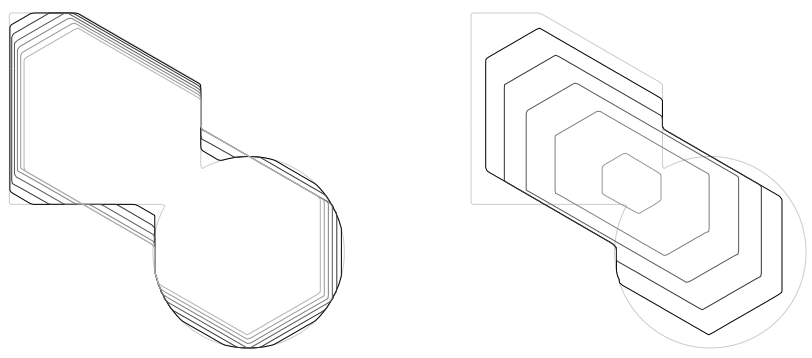

FIG. 4. The evolution at iterations $1,4,8,12,16,20$ and 20, 40, 60, 80, 100 (hexagonal Wulff shape).

introduction, it is likely that the consistency result in [1] yields the convergence of our algorithm to the generalized motion defined in [33].

The initial curve is the same as in Figure 2 Figure 3 shows the evolution at different times with a square Wulff shape (the initial curve is also plotted in light grey). Figure 4 shows the evolution with a hexagonal Wulff shape. Again, in both cases the size of the grid is $150 \times 150$.

\section{A. Some properties of the minimizer of (3)}

We state and prove in this section some properties of the solution of (3). All these properties also hold in the anisotropic case, for the solution of $(15)$, with identical proofs.

LEMmA A.1 Let $\bar{w}$ be the (unique) solution of (3). Then, for any $t>0, \bar{w} \wedge t$ is also the unique solution of

$$
\min _{w \leqslant t \text { a.e. }} \int_{\Omega} \frac{\left(w(x)-d_{E}(x)\right)^{2}}{2 h}+J(w) .
$$

Proof. First, we notice that for every $t$ and $w, \partial J(w) \subset \partial J(w \wedge t)$ : in the proof of Proposition 2.2. it is established that if $p \in \partial J(w)$, then $p \in \partial J\left(\mathbf{1}_{\{w>t\}}\right)$ for a.e. $t \in \mathbb{R}$. Now,

$$
J(w \wedge t)=\int_{-\infty}^{t} J(\{w>s\}) \mathrm{d} s=\int_{-\infty}^{t} \int_{\Omega} p(x) \mathbf{1}_{\{w>s\}}(x) \mathrm{d} x \mathrm{~d} s=\int_{\Omega} p(x)(w(x) \wedge t) \mathrm{d} x .
$$

We deduce that $p \in \partial J(w \wedge t)$.

Since $-\left(\bar{w}-d_{E}\right) / h \in \partial J(\bar{w}) \subset \partial J(\bar{w} \wedge t)$, for all $w$ we have

$$
\begin{aligned}
J(w) & \geqslant J(\bar{w} \wedge t)-\int_{\Omega} \frac{\bar{w}-d_{E}}{h}(w-\bar{w} \wedge t) \\
& =J(\bar{w} \wedge t)-\int_{\Omega} \frac{\bar{w} \wedge t-d_{E}}{h}(w-\bar{w} \wedge t)-\int_{\Omega} \frac{\bar{w}-\bar{w} \wedge t}{h}(w-\bar{w} \wedge t) \\
& =J(\bar{w} \wedge t)-\int_{\Omega} \frac{\bar{w} \wedge t-d_{E}}{h}(w-\bar{w} \wedge t)-\int_{\{\bar{w}>t\}} \frac{\bar{w}-t}{h}(w-t) .
\end{aligned}
$$

If $w \leqslant t$ a.e. in $\Omega$, then $-\int_{\{\bar{w}>t\}}(\bar{w}-t)(w-t) \geqslant 0$, hence

$$
J(w) \geqslant J(\bar{w} \wedge t)-\int_{\Omega} \frac{\bar{w} \wedge t-d_{E}}{h}(w-\bar{w} \wedge t) .
$$


As $\int_{\Omega}\left(w-d_{E}\right)^{2} \geqslant \int_{\Omega}\left(\bar{w} \wedge t-d_{E}\right)^{2}+2 \int_{\Omega}\left(\bar{w} \wedge t-d_{E}\right)(w-\bar{w} \wedge t)$, we obtain the assertion.

Corollary A.2 Let $E \subset \subset \Omega \subseteq \Omega^{\prime}$ and let $w$ be the minimizer of 3 in $\Omega$ while $w^{\prime}$ is the minimizer of $(3)$ in $\Omega^{\prime}$. Let $\delta=\operatorname{dist}(E, \partial \Omega)=\min _{\partial \Omega} d_{E}>0$ and assume that for some $t<\delta$, $\{w<t\} \subset \subset \Omega$. Then $w \wedge t=w^{\prime} \wedge t$.

Proof. Let $\widetilde{w}$ be the function given by $w \wedge t$ in $\Omega$ and $t$ in $\Omega \backslash \Omega^{\prime}$. We wish to show that $\widetilde{w}=w^{\prime} \wedge t$. We have

$$
\begin{aligned}
J\left(\tilde{w} ; \Omega^{\prime}\right)+\int_{\Omega^{\prime}} & \frac{\left(\widetilde{w}(x)-d_{E}(x)\right)^{2}}{2 h} \mathrm{~d} x \\
& =J(w \wedge t ; \Omega)+\int_{\Omega} \frac{\left(w(x) \wedge t-d_{E}(x)\right)^{2}}{2 h} \mathrm{~d} x+\int_{\Omega^{\prime} \backslash \Omega} \frac{\left(t-d_{E}(x)\right)^{2}}{2 h} \mathrm{~d} x \\
& \leqslant J\left(w^{\prime} \wedge t ; \Omega\right)+\int_{\Omega} \frac{\left(w^{\prime}(x) \wedge t-d_{E}(x)\right)^{2}}{2 h} \mathrm{~d} x+\int_{\Omega^{\prime} \backslash \Omega} \frac{\left(t-d_{E}(x)\right)^{2}}{2 h} \mathrm{~d} x
\end{aligned}
$$

where the second inequality follows from Lemma A.1 (in $\Omega$ ). Now, on $\Omega^{\prime} \backslash \Omega, d_{E} \geqslant \delta \geqslant t$, so that $\left(t-d_{E}(x)\right)^{2} \leqslant\left(w^{\prime}(x) \wedge t-d_{E}(x)\right)^{2}$ a.e. in $\Omega^{\prime} \backslash \Omega$. Also, $J\left(w^{\prime} \wedge t ; \Omega\right) \leqslant J\left(w^{\prime} \wedge t ; \Omega^{\prime}\right)$. We deduce that

$$
J\left(\tilde{w} ; \Omega^{\prime}\right)+\int_{\Omega^{\prime}} \frac{\left(\tilde{w}(x)-d_{E}(x)\right)^{2}}{2 h} \mathrm{~d} x \leqslant J\left(w^{\prime} \wedge t ; \Omega^{\prime}\right)+\int_{\Omega^{\prime}} \frac{\left(w^{\prime}(x) \wedge t-d_{E}(x)\right)^{2}}{2 h} \mathrm{~d} x,
$$

and by Lemma A.1 (in $\left.\Omega^{\prime}\right)$ it follows that $\widetilde{w}=w^{\prime} \wedge t$.

REMARK A.3 Taking $t=0$ in Corollary A.2 we deduce that as soon as $T_{h}(E) \subset \subset \Omega$, it is the same set, whether computed relative to $\Omega$ or to $\Omega^{\prime} \supseteq \Omega$ (that is, $\{w<0\}=\left\{w^{\prime}<0\right\}$ ).

Proposition A.4 Assume $\Omega$ is the torus $\mathbb{T}^{N}=(\mathbb{R} / \mathbb{Z})^{N}$. Then the solution $w$ of 3 is Lipschitz. In fact, $|\nabla w| \leqslant 1$ a.e. in $\Omega$.

Proof. Consider a sequence $\psi_{n}: \mathbb{R}^{N} \rightarrow[0,+\infty)$ of smooth convex Lagrangians such that $\left(D^{2} \psi_{n}(p) \xi\right) \cdot \xi \geqslant(1 / n)|\xi|^{2}$ for every $p, \xi \in \mathbb{R}^{N}, \psi_{n}(p) \geqslant|p|$ for each $p$ and $n$, and $\psi_{n}(p) \rightarrow|p|$ as $n \rightarrow \infty$ (locally uniformly). One shows that the solutions $w_{n}$ of

$$
\min _{w \in H^{1}(\Omega)} \int_{\Omega} \frac{\left(w(x)-d_{E}(x)\right)^{2}}{2 h} \mathrm{~d} x+\int_{\Omega} \psi_{n}(\nabla w(x)) \mathrm{d} x
$$

converge (at least, weakly in $L^{2}(\Omega)$ ) to the solution $w$ of 3 as $n \rightarrow \infty$. Indeed, if $w$ is the weak $L^{2}$-limit of a subsequence of $w_{n}$ (still denoted $\left.w_{n}\right)$, and $v \in C^{\infty}(\Omega)$, one has

$$
\begin{aligned}
\int_{\Omega} \frac{\left(w-d_{E}\right)^{2}}{2 h}+J(w) & \leqslant \liminf _{n \rightarrow \infty} \int_{\Omega} \frac{\left(w_{n}-d_{E}\right)^{2}}{2 h}+J\left(w_{n}\right) \\
& \leqslant \liminf _{n \rightarrow \infty} \int_{\Omega} \frac{\left(w_{n}-d_{E}\right)^{2}}{2 h}+\int_{\Omega} \psi_{n}\left(\nabla w_{n}\right) \\
& \leqslant \liminf _{n \rightarrow \infty} \int_{\Omega} \frac{\left(v-d_{E}\right)^{2}}{2 h}+\int_{\Omega} \psi_{n}(\nabla v)=\int_{\Omega} \frac{\left(v-d_{E}\right)^{2}}{2 h}+\int_{\Omega}|\nabla v| .
\end{aligned}
$$


But since any $v \in B V(\Omega)$ can be approximated by functions $v_{j} \in C^{\infty}(\Omega)$, with $\int_{\Omega}\left|\nabla v_{j}\right| \rightarrow J(v)$ as $j \rightarrow \infty$ (see [40]), this shows that $w$ solves $(3)$.

Now, the Euler equation for 21 is

$$
w_{n}(x)-d_{E}(x)-h \operatorname{div} \nabla \psi_{n}\left(\nabla w_{n}(x)\right)=0 .
$$

Differentiating in the direction $e \in \mathbb{S}^{N-1}$ (we set $\partial_{e}=e \cdot \nabla$ ), we find

$$
\partial_{e} w_{n}(x)-\partial_{e} d_{E}(x)-h \operatorname{div} D^{2} \psi_{n}\left(\nabla w_{n}(x)\right) \nabla\left(\partial_{e} w_{n}\right)(x)=0 .
$$

Since $D^{2} \psi_{n}\left(\nabla w_{n}(x)\right) \geqslant(1 / n) I$ for a.e. $x \in \Omega$, we deduce by the maximum principle that $\left\|\partial_{e} w_{n}\right\|_{L^{\infty}(\Omega)} \leqslant\left\|\partial_{e} d_{E}\right\|_{L^{\infty}(\Omega)}$. Since this is true for any $e \in \mathbb{S}^{N-1}$, it shows that $\left|\nabla w_{n}\right| \leqslant 1$ a.e. in $\Omega$. Hence also $|\nabla w| \leqslant 1$. (Notice that $w_{n}$ converges uniformly to $w$.)

REMARK A.5 We had to assume $\Omega$ is a torus in order to get rid of the problems at the boundary in the proof. However, we believe that $w$ should be Lipschitz continuous as soon as $\Omega$ is convex (in which case we probably still have $|\nabla w| \leqslant 1$ a.e.) or $\partial \Omega$ is smooth enough. See also Corollary A.7.

REMARK A.6 In the anisotropic case (if $w$ solves (15)), one deduces in the same way that $\varphi^{\circ}(\nabla w) \leqslant 1$ a.e. in the torus.

Corollary A.7 Assume $\Omega$ is the cube $(0,1)^{N} \subset \mathbb{R}^{N}$ (or, more generally, of the form $\left(0, L_{1}\right) \times$ $\cdots \times\left(0, L_{N}\right)$ ), and $w_{h}$ solves $(3)$. Then $\left|\nabla w_{h}\right| \leqslant 1$ a.e. in $\Omega$. In particular, as $h \rightarrow 0, w_{h}$ goes uniformly to $d_{E}$ in $\Omega$.

Proof. The problem in a hypercube is equivalent to the problem in a torus of period twice that of the cube. Indeed, we can first symmetrize the data $\left(d_{E}\right)$ across the boundaries of $\Omega=(0,1)^{N}$, and then periodize it (with period 2 in each direction). It is straightforward to check that the solution of the periodized problem is the same as the original function $w_{h}$. The conclusion follows by AscoliArzelà's theorem. In the anisotropic case again we get $\varphi^{\circ}\left(\nabla w_{h}\right) \leqslant 1$ a.e. in $\Omega$.

REMARK A.8 We immediately see that if $E \subset \subset \Omega$, then in this case $T_{h}(E) \subset \subset \Omega$ for $h$ small enough, since $w_{h}$ has to be larger than $\operatorname{dist}(E, \partial \Omega) / 2$ for $h$ small enough in a neighbourhood of $\partial \Omega$

Corollary A.9 Assume $E \subset \subset \Omega$ and $\{w<t\} \subset \subset \Omega$ for some $t<\delta=\operatorname{dist}(E, \partial \Omega)$. Then $|\nabla w| \leqslant 1$ a.e. in $\{w<t\}$.

Proof. Consider $L$ large enough so that $\Omega \subset \subset(-L, L)^{N}$. Let $\mathbb{T}_{2 L}^{N}=(\mathbb{R} /(2 L \mathbb{Z}))^{N}$ be the torus of period $2 L$, and define $\widetilde{\Omega}$ as the periodization of $\Omega$ in $\mathbb{T}_{2 L}^{N}\left(x \in \widetilde{\Omega}\right.$ iff $\pi^{-1}(x) \cap \Omega \neq \emptyset$, where $\pi: \mathbb{R}^{N} \rightarrow \mathbb{T}_{2 L}^{N}$ is the canonical projection). Define $\widetilde{E} \subset \widetilde{\Omega}$ in the same way.

It is clear that $\widetilde{w}: \widetilde{\Omega} \rightarrow \mathbb{R}$ defined by $\widetilde{w}(\pi(x))=w(x)$ when $x \in \Omega$ is the unique solution of problem $\sqrt{3}$ in $\widetilde{\Omega}$, with $E$ replaced with $\widetilde{E}$. By Corollary A.2 $\widetilde{w} \wedge t=w^{\prime} \wedge t$, where $w^{\prime}$ is the solution of the minimization problem A.2 but this time in the whole torus $\mathbb{T}_{2 L}^{N}$. By the previous proposition, $\left|\nabla w^{\prime}\right| \leqslant 1$ a.e. in the torus. Hence also $|\nabla \widetilde{w}| \leqslant 1$ a.e. in $\{\widetilde{w}<t\}$, or, which is the same, $|\nabla w| \leqslant 1$ a.e. in $\{w<t\}$. 


\section{Acknowledgements}

The author would like to thank Guy Barles, Giovanni Bellettini and Matteo Novaga for many interesting discussions. Also, the anonymous referees made helpful comments and mentioned a few appropriate references. This work was partially supported by the European TMR Network "Viscosity Solutions and Their Applications".

Note added in proof. It has been observed by Pierre Cardaliaguet that the construction in this paper, together with the semigroup property of the generalized mean curvature flow, implies that under the same assumptions as in Theorem 4, the perimeter of $E(t)$ is nonincreasing (see [31, Thm. 6.3]).

\section{REFERENCES}

1. Almgren, F. \& TAYloR, J. E. Flat flow is motion by crystalline curvature for curves with crystalline energies. J. Diff. Geom. 42 (1995), 1-22. Zbl 0867.58020 MR 96h:58034

2. Almgren, F., TAYlOR, J. E., \& WANG, L.-H. Curvature-driven flows: a variational approach. SIAM J. Control Optim. 31 (1993), 387-438. Zbl 0783.35002 MR 94h:58067

3. Ambrosio, L. Movimenti minimizzanti. Rend. Accad. Naz. Sci. XL Mem. Mat. Appl. (5) 19 (1995), 191-246. Zbl 0957.49029 MR 97c:49044

4. Angenent, S. \& Gurtin, M. E. Anisotropic motion of a phase interface. Well-posedness of the initial value problem and qualitative properties of the interface. J. Reine Angew. Math. 446 (1994), 1-47. Zbl 0784.35124 MR 95c:35273

5. Angenent, S. \& GURTin, M. E. Multiphase thermomechanics with interfacial structure. II. Evolution of an isothermal interface. Arch. Rat. Mech. Anal. 108(1989), 323-391. Zbl 0723.73017 MR 91d:73004

6. BARles, G. Solutions de viscosité des équations de Hamilton-Jacobi. Math. Appl. 17, Springer, Paris (1994). Zbl 0819.35002 MR 2000b:49054

7. Barles, G. \& Georgelin, C. A simple proof of convergence for an approximation scheme for computing motions by mean curvature. SIAM J. Numer. Anal. 32 (1995), 484-500. Zbl 0831.65138 MR 96c:65140

8. BARles, G., Soner, H. M., \& Souganidis, P. E. Front propagation and phase field theory. SIAM J. Control Optim. 31 (1993), 439-469. Zbl 0785.35049 MR 94c:35005

9. BARles, G. \& Souganidis, P. E. A new approach to front propagation problems: theory and applications. Arch. Rat. Mech. Anal. 141 (1998), 237-296. Zbl 0904.35034 MR 99c:35106

10. Bellettini, G., Goglione, R., \& Novaga, M. Approximation to driven motion by crystalline curvature in two dimensions. Adv. Math. Sci. Appl. 10 (2000), 467-493. Zbl 0979.53075 MR 2001i:53109

11. Bellettini, G. \& Novaga, M. Barriers for a class of geometric evolution problems. Atti Accad. Naz. Lincei Cl. Sci. Fis. Mat. Natur. Rend. Lincei (9) Mat. Appl. 8 (1997), 119-128. Zbl 0990.35070 MR 98i:49047

12. Bellettini, G. \& Novaga, M. Minimal barriers for geometric evolutions. J. Differential Equations 139 (1997), 76-103. Zbl 0882.35028 MR 98j:35083

13. Bellettini, G. \& Novaga, M. Comparison results between minimal barriers and viscosity solutions for geometric evolutions. Ann. Scuola Norm. Sup. Pisa Cl. Sci. (4) 26 (1998), 97-131. Zbl 0904.35041 MR 99i:35081

14. Bellettini, G. \& Novaga, M. Some aspects of De Giorgi's barriers for geometric evolutions. Calculus of Variations and Partial Differential Equations (Pisa, 1996), Springer, Berlin (2000), 115-151. Zbl 0957.35080 MR 1757698

15. Bellettini, G., Novaga, M., \& Paolini, M. Facet-breaking for three-dimensional crystals evolving by mean curvature. Interfaces Free Bound. 1 (1999), 39-55. Zbl 0934.49023 MR 2003i:53099 
16. Bellettini, G. \& Paolini, M. Comparison theorems for various notions of curvature-driven motion. Atti Accad. Naz. Lincei Cl. Sci. Fis. Mat. Natur. Rend. Lincei (9) Mat. Appl. 6 (1995), 45-54. Zbl 0834.35062 MR 96h:35083

17. Bellettini, G. \& Paolini, M. Some results on minimal barriers in the sense of De Giorgi applied to driven motion by mean curvature. Rend. Accad. Naz. Sci. XL Mem. Mat. Appl. (5) 19 (1995), 43-67; Errata, 26 (2002), 161-165. Zbl 0944.53039 MR 97b:49038 MR 1976356

18. Bellettini, G. \& Paolini, M. Anisotropic motion by mean curvature in the context of Finsler geometry. Hokkaido Math. J. 25 (1996), 537-566. Zbl 0873.53011 MR 97i:53079

19. BRAKKe, K. A. The Motion of a Surface by its Mean Curvature. Math. Notes 20, Princeton Univ. Press, Princeton, NJ (1978). Zbl 0386.53047 MR 82c:49035

20. CAO, F. Partial differential equations and mathematical morphology. J. Math. Pures Appl. (9) 77 (1998), 909-941. Zbl 0920.35040 MR 99m:35097

21. Chambolle, A. An algorithm for total variation minimization and applications. J. Math. Imaging Vision 20 (2004), 89-97.

22. Chen, Y.-G., Giga, Y., \& Goto, S. Uniqueness and existence of viscosity solutions of generalized mean curvature flow equations. J. Diff. Geom. 33 (1991), 749-786. Zbl 0696.35087 MR 93a:35093

23. CRandall, M. G., Ishit, H., \& LiOns, P.-L. User's guide to viscosity solutions of second order partial differential equations. Bull. Amer. Math. Soc. (N.S.) 27 (1992), 1-67. Zbl 0755.35015 MR 92j:35050

24. Ekeland, I. \& Temam, R. Convex Analysis and Variational Problems. North-Holland, Amsterdam (1976). Zbl 0322.90046 MR 57 \#3931b

25. Evans, L. C. Convergence of an algorithm for mean curvature motion. Indiana Univ. Math. J. 42 (1993), 533-557. Zbl 0802.65098 MR 95d:58023

26. Evans, L. C. \& Gariepy, R. F. Measure Theory and Fine Properties of Functions. CRC Press, Boca Raton, FL (1992). Zbl 0804.28001 MR 93f:28001

27. Evans, L. C., Soner, H. M., \& Souganidis, P. E. Phase transitions and generalized motion by mean curvature. Comm. Pure Appl. Math. 45 (1992), 1097-1123. Zbl 0801.35045 MR 93g:35064

28. Evans, L. C. \& Spruck, J. Motion of level sets by mean curvature. I. J. Diff. Geom. 33 (1991), 635-681. Zbl 0726.53029 MR 92h:35097

29. Evans, L. C. \& Spruck, J. Motion of level sets by mean curvature. II. Trans. Amer. Math. Soc. 330 (1992), 321-332. Zbl 0776.53005 MR 92f:58050

30. Evans, L. C. \& Spruck, J. Motion of level sets by mean curvature. III. J. Geom. Anal. 2 (1992), 121-150. Zbl 0768.53003 MR 93d:58044

31. Evans, L. C. \& Spruck, J. Motion of level sets by mean curvature. IV. J. Geom. Anal. 5 (1995), 77-114. Zbl 0829.53040 MR 96a:35077

32. Fierro, F., Goglione, R., \& Paolini, M. Numerical simulations of mean curvature flow in the presence of a nonconvex anisotropy. Math. Models Methods Appl. Sci. 8 (1998), 573-601. Zbl 0946.58014 MR 99k:58040

33. Giga, M.-H. \& GigA, Y. Generalized motion by nonlocal curvature in the plane. Arch. Rat. Mech. Anal. 159 (2001), 295-333. Zbl 1004.35075 MR 2002h:53117

34. Giga, Y. \& Goto, S. Geometric evolution of phase-boundaries. On the Evolution of Phase Boundaries (Minneapolis, MN, 1990-91), IMA Vol. Math. Appl. 43, Springer, New York (1992), 51-65. Zbl 0771.35027 MR 94g:35226

35. Giga, Y. \& Gurtin, M. E. A comparison theorem for crystalline evolution in the plane. Quart. Appl. Math. 54 (1996), 727-737. Zbl 0862.35047 MR 97m:80012

36. Giga, Y., Gurtin, M. E., \& Matias, J. On the dynamics of crystalline motions. Japan J. Indust. Appl. Math. 15 (1998), 7-50. MR 99h:73008

37. GIRÃO, P. M. Convergence of a crystalline algorithm for the motion of a simple closed convex curve by weighted curvature. SIAM J. Numer. Anal. 32 (1995), 886-899. Zbl 0830.65150 MR 96c:65144 
38. GiRÃo, P. M. \& KOHN, R. V. Convergence of a crystalline algorithm for the heat equation in one dimension and for the motion of a graph by weighted curvature. Numer. Math. 67 (1994), 41-70. Zbl 0791.65063 MR 94m:65138

39. GIRÃO, P. M. \& KoHN, R. V. The crystalline algorithm for computing motion by curvature. Variational Methods for Discontinuous Structures (Como, 1994), Progr. Nonlinear Differential Equations Appl. 25, Birkhäuser, Basel (1996), 7-18. Zbl 0869.65075 MR 97h:49053

40. Giusti, E. Minimal Surfaces and Functions of Bounded Variation. Birkhäuser, Basel (1984). Zbl 0545.49018 MR 87a:58041

41. Gurtin, M. E. Thermomechanics of Evolving Phase Boundaries in the Plane. Oxford Math. Monographs, Clarendon Press (1993). Zbl 0787.73004 MR 97k:73001

42. IshiI, H. A generalization of the Bence, Merriman and Osher algorithm for motion by mean curvature. Curvature Flows and Related Topics (Levico, 1994), GAKUTO Internat. Ser. Math. Sci. Appl. 5, Gakkōtosho, Tokyo (1995), 111-127. Zbl 0844.35043 MR 96m:35121

43. Ishit, H., Pires, G. E., \& Souganidis, P. E. Threshold dynamics type approximation schemes for propagating fronts. J. Math. Soc. Japan 51 (1999), 267-308. Zbl 0935.53006 MR 2000j:65016

44. LEONI, F. Convergence of an approximation scheme for curvature-dependent motions of sets. SIAM J. Numer. Anal. 39 (2001), 1115-1131. Zbl 1008.65067 MR 2002j:65093

45. Luckhaus, S. \& Sturzenhecker, T. Implicit time discretization for the mean curvature flow equation. Calc. Var. Partial Differential Equations 3 (1995), 253-271. Zbl 0821.35003 MR 97e:65085

46. Merriman, B., Bence, J. K., \& Osher, S. J. Diffusion generated motion by mean curvature. Computational Crystal Growers Workshop, J. E. Taylor (ed.), Amer. Math. Soc. (1992), 73-83.

47. Merriman, B., Bence, J. K., \& Osher, S. J. Motion of multiple functions: a level set approach. $J$. Comput. Phys. 112 (1994), 334-363. Zbl 0805.65090 MR 95a:65169

48. Osher, S. \& Sethian, J. A. Fronts propagating with curvature-dependent speed: algorithms based on Hamilton-Jacobi formulations. J. Comput. Phys. 79 (1988), 12-49. Zbl 0659.65132 MR 89h:80012

49. PaOlini, M. \& PASQuarelli, F. Numerical simulation of crystalline curvature flow in 3D by interface diffusion. Free Boundary Problems: Theory and Applications, II (Chiba, 1999), GAKUTO Internat. Ser. Math. Sci. Appl. 14, Gakkōtosho, Tokyo (2000), 376-389. Zbl 0979.53076 MR 2001i:74062

50. Rouy, E. \& Tourin, A. A viscosity solutions approach to shape-from-shading. SIAM J. Numer. Anal. 29 (1992), 867-884. Zbl 0754.65069 MR 93d:65019

51. RuUth, S. J. \& Merriman, B. Convolution-generated motion and generalized Huygens' principles for interface motion. SIAM J. Appl. Math. 60 (2000), 868-890. Zbl 0958.65021 MR 2001c:35107

52. Sethian, J. A. Fast marching methods. SIAM Rev. 41 (1999), 199-235. Zbl 0926.65106 MR 2000m:65125

53. TAYLOR, J. E. Motion of curves by crystalline curvature, including triple junctions and boundary points. Differential Geometry: Partial Differential Equations on Manifolds (Los Angeles, CA, 1990), Proc. Sympos. Pure Math. 54, Amer. Math. Soc., Providence, RI (1993), 417-438. Zbl 0823.49028 MR 94c:53012

54. TAYlor, J. E. Surface motion due to crystalline surface energy gradient flows. Elliptic and Parabolic Methods in Geometry (Minneapolis, MN, 1994), A. K. Peters, Wellesley, MA (1996), 145-162. Zbl 0915.49024 MR 97h:49054

55. Taylor, J. E., Cahn, J. W., \& Handwerker, C. A. Geometric models of crystal growth. Acta Metall. 40 (1992), 1443-1474.

56. TsitsikLIS, J. N. Efficient algorithms for globally optimal trajectories. IEEE Trans. Automat. Control 40 (1995), 1528-1538. Zbl 0831.93028 MR 96d:49039

57. Ziemer, W. P. Weakly Differentiable Functions. Springer, New York (1989). Zbl 0692.46022 MR 91e:46046 